\title{
Iterative methods for fixed point problems and generalized split feasibility problems in Banach spaces
}

\author{
Yanlai Song \\ College of Science, Zhongyuan University of Technology, 450007 Zhengzhou, China.
}

Communicated by X. Qin

\begin{abstract}
In this paper, we study the Halpern type iterative algorithm to approximate a common solution of fixed point problems of an infinite family of demimetric mappings and generalized split feasibility problems with firmly nonexpansive-like mappings in Banach spaces. We also prove strong convergence theorems for a common solution of the above-said problems by the proposed iterative algorithm and discuss some applications of our results. The methods in this paper are novel and different from those given in many other paper. And the results are the extension and improvement of the recent results in the literature.
\end{abstract}

Keywords: Banach space, generalized split feasibility problem, fixed point, metric resolvent, demimetric mapping.

2010 MSC: 47H09, 47H10, $49 \mathrm{H} 17$.

(C)2018 All rights reserved.

\section{Introduction}

Let $\mathrm{H}$ be a real Hilbert space, $\mathrm{C}$ a nonempty closed convex subset of $\mathrm{H}, \mathrm{T}$ a mapping on $\mathrm{C}$, and $\mathrm{F}(\mathrm{T}):=\{x \in \mathrm{C}: \mathrm{T} x=\mathrm{x}\}$.

Definition 1.1. A mapping $\mathrm{T}: \mathrm{C} \rightarrow \mathrm{H}$ is said to be:

(1) a k-strict pseudo-contraction if there exists $k \in[0,1)$ such that

$$
\|\mathrm{T} x-\mathrm{T} y\|^{2} \leqslant\|x-y\|^{2}+k\|x-T x-(y-T y)\|^{2}, \quad \forall x, y \in C ;
$$

(2) a 2-generalized hybrid mapping if there exist $\delta_{1}, \delta_{2}, \epsilon_{1}, \epsilon_{2} \in \mathbb{R}$ such that

$$
\begin{aligned}
& \delta_{1}\left\|\mathrm{~T}^{2} x-\mathrm{T} y\right\|^{2}+\delta_{2}\|\mathrm{~T} x-\mathrm{T} y\|^{2}+\left(1-\delta_{1}-\delta_{2}\right)\|x-\mathrm{T} y\|^{2} \\
& \quad \leqslant \epsilon_{1}\left\|\mathrm{~T}^{2} x-y\right\|^{2}+\epsilon_{2}\|\mathrm{~T} x-y\|^{2}+\left(1-\epsilon_{1}-\epsilon_{2}\right)\|x-y\|^{2}, \quad \forall x, y \in C .
\end{aligned}
$$

We know that the class of 2-generalized hybrid mappings contains the classes of nonexpansive mappings, nonspreading mappings, hybrid mappings, and generalized hybrid mappings in a Hilbert space;

Email address: songyanlai2009@163.com (Yanlai Song)

doi: $10.22436 /$ jnsa.011.02.03

Received: 2017-08-25 Revised: 2017-11-23 Accepted: 2017-12-06 
see [13, 31]. In general, 2-generalized hybrid mappings are not continuous; see [12]. Hence, the class of k-strict pseudo-contractions does not contain the class of 2-generalized hybrid mappings by the fact that k-strict pseudo-contractions are continuous. We give an example for a 2-generalized hybrid mapping, but not a k-strict pseudo-contraction.

Example 1.2 ([17]). Let $S:[0,2] \rightarrow \mathbb{R}$ be defined as

$$
S x= \begin{cases}0, & x \in[0,2) \\ 1, & x=2\end{cases}
$$

Then $S$ is a 2-generalized hybrid mapping and $F(S)=\{0\}$. However, it is not a k-strict pseudo-contraction.

Certainly, the class of 2-generalized hybrid mappings does not contain the class of k-strict pseudocontractions. We give an example for a k-strict pseudo-contraction which is not a 2-generalized hybrid mapping.

Example 1.3. Let $S:[-2,2] \rightarrow \mathbb{R}$ be defined as

$$
S x=-3 x .
$$

Then $S$ is a k-strict pseudo-contraction, but not a 2-generalized hybrid mapping (check for instance the condition of 2-generalized hybrid mapping for $x=0$ and $y=-1$ ).

Recently, Takahashi [25] introduced a broad class of nonlinear mappings in a Hilbert space called k-demimetric mapping. This class of mappings contains the classes of 2-generalized hybrid mappings, k-strict pseudo-contractions, firmly quasi-nonexpansive mappings, and quasi-nonexpansive mappings.

Definition 1.4. Let $\mathrm{H}$ be a Hilbert space and let $\mathrm{C}$ be a nonempty, closed, and convex subset of $\mathrm{H}$. Let $k \in(-\infty, 1)$. A mapping $T: C \rightarrow H$ with $F(T) \neq \emptyset$ is called $k$-demimetric if, for any $x \in C$ and $q \in F(T)$,

$$
\langle x-\mathrm{q}, x-\mathrm{T} x\rangle \geqslant \frac{1-\mathrm{k}}{2}\|x-\mathrm{T} x\|^{2} .
$$

It is clear that (1.1) is equivalent to the following:

$$
\|T x-q\|^{2} \leqslant\|x-q\|^{2}+k\|x-T x\|^{2} .
$$

We give an example of a k-demimetric mapping which is not pseudo-contractive, hence is not strictly pseudo-contractive.

Example 1.5. ([10]) Let $H$ be the real line and $C=[-1,1]$. Define $T$ on $C$ by $T(x)=\frac{2}{3} x \sin \frac{1}{x}$ if $x \neq 0$ and $T(0)=0$. Clearly, 0 is the only fixed point of $T$. Also, for $x \in C,|T(x)-0|^{2}=|T(x)|^{2}=\left|\frac{2}{3} x \sin \frac{1}{x}\right|^{2} \leqslant$ $\left|\frac{2 x}{3}\right|^{2} \leqslant|x|^{2} \leqslant|x-0|^{2}+k|T(x)-x|^{2}$ for any $k \in[0,1)$. Thus $T$ is demimetric. We show that $T$ is not pseudo-contractive. Let $x=\frac{2}{\pi}$ and $y=\frac{2}{3 \pi}$. Then $|T(x)-T(y)|=\frac{256}{81 \pi^{2}}$. However,

$$
|x-y|^{2}+|(I-T) x-(I-T) y|^{2}=\frac{160}{81 \pi^{2}} \text {. }
$$

Let $E$ be a smooth Banach space and $C$ a nonempty subset of $E$. A mapping $S: C \rightarrow E$ is said to be a firmly nonexpansive-like mapping (or a mapping of type $(\mathrm{P})$ (see [4])) if

$$
\langle S x-S y, J(x-S x)-J(y-S y)\rangle \geqslant 0, \quad \forall x, y \in C .
$$

If $E$ is a Hilbert space, then $T$ is firmly nonexpansive-like if and only if it is firmly nonexpansive, i.e., $\|T x-T y\| \leqslant\langle T x-T y, x-y\rangle$ for all $x, y \in C$. We give two examples of the firmly nonexpansive-like mapping as following.

Example 1.6. Let $E$ be a smooth, strictly convex, and reflexive Banach space and $C$ a nonempty closed convex subset of $\mathrm{E}$. Then the metric projection $\mathrm{P}_{\mathrm{C}}$ is a firmly nonexpansive-like mapping.

Example 1.7. Let $E$ be a smooth, strictly convex, and reflexive Banach space, $A \subset E \times E^{*}$ a monotone operator, and $r>0$. Then the metric resolvent $J_{r}$ of $A$ is a firmly nonexpansive-like mapping. 
The class of firmly nonexpansive-like mappings plays an important role in nonlinear analysis and optimization. The problem of finding the fixed points for such mappings is the subject of current interest in functional analysis. It turns out that the fixed point theory for such mappings can be applied to several nonlinear problems such as zero point problems for monotone operators, convex feasibility problems, convex minimization problems, variational inequality, and equilibrium problems, and so on; see [2-4] for more details.

On the other hand, we also have the split feasibility problem (SFP). The SFP in the setting of finitedimensional Hilbert spaces was first introduced by Censor and Elfving [7] for modelling inverse problems which arises from phase retrievals and in medical image reconstruction [6]. Since then, a lot of work has been done on finding a solution of SFP. Recently, it is found that the SFP can also be applied to study the intensity-modulated radiation therapy; see, for example, $[8,18]$ and the references therein.

At the same time, to construct a mathematical model which is as close as possible to a real complex problem, we often have to use more than one constraint. Solving such problems, we have to obtain some solution which is simultaneously the solution of two or more subproblems or the solution of one subproblem on the solution set of another subproblem. These subproblems can be given, for example, by two or more different SFPs or two or more different fixed point problems. It is natural to construct a unified approach for these problems. In this direction, several authors have introduced some iterative schemes for finding a common element of the set of solutions of the SFPs and the set of fixed points of nonlinear mappings; see, for example, $[9,11,22]$ and the references therein.

In order to finding a common fixed point of an infinite family of demimetric mappings in a Hilbert space, Akashi and Takahashi [1] introduced the following hybrid method in mathematical programming without assuming that demimetric mappings are commutative:

$$
\left\{\begin{array}{l}
z_{n}=\sum_{j=1}^{\infty} \xi_{n}\left(\left(1-\lambda_{n}\right) I+\lambda_{n} T_{j}\right) x_{n} \\
x_{n+1}=P_{C}\left(\alpha_{n} x_{n}+\left(1-\alpha_{n}\right) z_{n}\right)
\end{array}\right.
$$

where $\left\{T_{j}\right\}_{j=1}^{\infty}: C \rightarrow H$ is an infinite family of $k_{i}$-demimetric and demiclosed mappings. Then they obtained a weak convergence theorem under some mild restrictions on the parameters.

On the other hand, Takahashi and Yao [27] considered the following split common null point problem in Banach spaces: Given set-valued mappings $\mathrm{A}: \mathrm{H} \rightarrow 2^{\mathrm{H}}, \mathrm{B}: \mathrm{E} \rightarrow 2^{\mathrm{E}^{*}}$, respectively, and bounded linear operator $\mathrm{T}: \mathrm{H} \rightarrow \mathrm{E}$, the split common null point problem [27] is to find a point $z \in \mathrm{H}$ such that

$$
z \in \mathrm{A}^{-1} 0 \bigcap \mathrm{T}^{-1}\left(\mathrm{~B}^{-1} 0\right) \neq \emptyset
$$

where $A^{-1} 0$ and $B^{-1} 0$ are null point sets of $A$ and $B$, respectively. In order to finding a solution of the split common null point problem in Banach spaces, they also introduced the following hybrid method in mathematical programming:

$$
\left\{\begin{array}{l}
z_{n}=J_{\lambda_{n}}\left(x_{n}-\lambda_{n} T^{*} J_{E}\left(T x_{n}-Q_{\mu_{n}} T x_{n}\right)\right), \\
y_{n}=\alpha_{n} z_{n}+\left(1-\alpha_{n}\right) x_{n} \\
C_{n}=\left\{z \in H:\left\|y_{n}-u\right\| \leqslant\left\|x_{n}-u\right\|,\right. \\
Q_{n}=\left\{z \in H:\left\langle x_{n}-z, x_{1}-x_{n}\right\rangle \geqslant 0\right\} \\
x_{n+1}=P_{C_{n} \cap Q_{n}} x_{1}, \quad \forall n \in \mathbb{N},
\end{array}\right.
$$

where $J_{\lambda}$ is the resolvent of $A$ for $\lambda>0, Q_{\mu}$ is the metric resolvent of $B$ for $\mu>0, T: H \rightarrow E$ is a bounded linear operator such that $T \neq 0$ and $T^{*}$ is the adjoint operator of $T$. Then they obtained a strong convergence theorem under some mild restrictions on the parameters. However, we find that the sequence generated by the above algorithm is difficult to compute because it involves projecting $x_{0}$ onto the intersection of closed convex sets $C_{n}$ and $Q_{n}$ for each $n \geqslant 1$.

Motivated and inspired by Akashi and Takahashi [1] and Takahashi and Yao [27], we analyze the Halpern type iterative algorithm for finding a common solution of fixed point problems of an infinite 
family of demimetric mappings and generalized split feasibility problems with firmly nonexpansivelike mappings in Banach spaces. We prove the strong convergence of the proposed algorithm without requiring the complex computation of $C_{n} \cap Q_{n}$ for each $n \geqslant 1$. Finally, we apply our convergence results to the convex minimization problem. Our results improve essentially the corresponding results in $[1,27]$. Further, some other results are also improved; see $[3,15,30]$.

\section{Preliminaries}

Throughout this paper, we denote $E$ the real Banach space, $E^{*}$ the dual of $E$, I the identity mapping on $E, H$ the real Hilbert space, $\mathbb{R}$ the set of real numbers, and $\mathbb{N}$ the set of positive integers. The expressions $x_{n} \rightarrow x$ and $x_{n} \rightarrow x$ denote the strong and weak convergence of the sequence $\left\{x_{n}\right\}$, respectively. The (normalized) duality mapping $J$ from $E$ to $E^{*}$ is defined by

$$
J x=\left\{x^{*} \in E^{*}:\left\langle x, x^{*}\right\rangle=\|x\|^{2}=\left\|x^{*}\right\|^{2}\right\}
$$

for all $x \in E$, where $\langle\cdot, \cdot\rangle$ denotes the duality product. If $E$ is a Hilbert space, then $J=I$, where $I$ is the identity mapping on $\mathrm{H}$.

The norm of a Banach space $E$ is said to be Gâteaux differentiable if the limit

$$
\lim _{t \rightarrow 0} \frac{\|x+t y\|-\|x\|}{t}
$$

exists for all $x, y$ on the unit sphere $S(E)=\{x \in E:\|x\|=1\}$. In this case, we say that $E$ is smooth.

The norm of $E$ is said to be uniformly Gâteaux differentiable if for each $y \in S(E)$, the limit (2.1) is attained uniformly for $x \in S(E)$. The norm of $E$ is said to be Fréchet differentiable if for each $x \in S(E)$, the limit (2.1) is attained uniformly for $y \in S(E)$. The norm of $E$ is said to be uniformly Fréchet differentiable if the limit (2.1) is attained uniformly for $x, y \in S(E)$. In this case $E$ is said to be uniformly smooth. It is known that

- if $\mathrm{E}$ is smooth, then the duality mapping J is single-valued;

- if the norm of $E$ is uniformly Gâteaux differentiable, then $J$ is uniformly norm-to-weak* continuous on each bounded subset of $E$;

- if the norm of $E$ is Fréchet differentiable, then J is norm-to-norm continuous;

- if $E$ is uniformly smooth, then $J$ is uniformly norm-to-norm continuous on each bounded subset of E;

see $[23,24]$ for more details.

A Banach space $E$ is said to be strictly convex if $\|x-y\|<2$ whenever $x, y \in S(E)$ and $x \neq y$. It is known that if $E$ is strictly convex, then the duality mapping $J$ is injective, that is, $x, y \in E$ and $x \neq y$ imply $J x \cap J y=\emptyset$. A Banach space $E$ is said to be uniformly convex if $\left\|x_{n}-y_{n}\right\| \rightarrow 0$ whenever $\left\{x_{n}\right\}$ and $\left\{y_{n}\right\}$ are sequences in $S(E)$ and $\left\|x_{n}+y_{n}\right\| \rightarrow 2$. It is known that if $E$ is uniformly convex, then

- $E$ is strictly convex and reflexive;

- E has the Kadec-Klee property, that is, a sequence $\left\{x_{n}\right\}$ in E converges strongly to $x$ whenever $x_{n} \rightarrow x$ and $\left\|x_{n}\right\| \rightarrow\|x\| ;$

see $[23,24]$ for more details.

We know that $E$ is reflexive if and only if $J$ is surjective. Therefore, if $E$ is a smooth, strictly convex and reflexive Banach space, then $\mathrm{J}$ is a single-valued bijection and in this case, the inverse mapping $\mathrm{J}^{-1}$ coincides with the duality mapping $\mathrm{J}^{*}$ on $\mathrm{E}^{*}$.

Let $E$ be a Banach space and let $A$ be a mapping of $E$ into $2^{E^{*}}$. The effective domain of $A$ is denoted by $\operatorname{dom}(A)$, that is, $\operatorname{dom}(A)=\{x \in E: A x \neq \emptyset\}$. A multi-valued mapping $A$ on $E$ is said to be monotone if $\left\langle x-y, u^{*}-v^{*}\right\rangle \geqslant 0$ for all $x, y \in \operatorname{dom}(A), u^{*} \in A x$, and $v^{*} \in A y$. A monotone operator $A$ on $E$ is said 
to be maximal if its graph is not properly contained in the graph of any other monotone operator on $E$. Suppose $E$ is a smooth, strictly convex, and reflexive Banach space and $A$ is a monotone operator. Then it is known that the single-valued mapping $J_{r}:=\left(I+r J^{-1} A\right)^{-1}$ is well-defined for all $r>0$; see [5]. Such $\mathrm{J}_{r}, \mathrm{r}>0$ are called the metric resolvent of $A$. It is also known that if $A$ is a maximal monotone operator, then

$$
\operatorname{ran}\left(I+r J^{-1} A\right)=E
$$

for all $r>0$, where $\operatorname{ran}\left(I+r J^{-1} A\right)$ is the range of $\left(I+r J^{-1} A\right)$; see [4]. The set of null points of $A$ is defined by $A^{-1} 0=\{z \in E: 0 \in A z\}$. We know that $A^{-1} 0$ is closed and convex and $F\left(J_{r}\right)=A^{-1} 0$; see [4].

Let $H$ be a Hilbert space and let $A$ be a maximal monotone operator on a Hilbert space $H$. The metric resolvent $J_{r}$ of $A$ is called the resolvent of $A$ simply. It is known that the resolvent $J_{r}$ of $A$ for any $r>0$ is firmly nonexpansive, i.e.,

$$
\left\|J_{r} x-J_{r} y\right\|^{2} \leqslant\left\langle x-y, J_{r} x-J_{r} y\right\rangle, \quad \forall x, y \in H .
$$

Lemma 2.1. In a Hilbert space $\mathrm{H}$, it holds for all $\mathrm{x}, \mathrm{y} \in \mathrm{H}$ and $\lambda \in[0,1]$ that

$$
\|\lambda x+(1-\lambda) y\|^{2}=\lambda\|x\|^{2}+(1-\lambda)\|y\|^{2}-\lambda(1-\lambda)\|x-y\|^{2},
$$

which can be extended to the more general situation: for all $x_{1}, x_{2}, \ldots, x_{n} \in H, \lambda_{i} \in[0,1]$, and $\sum_{i=1}^{n} \lambda_{i}=1$, we have

$$
\left\|\lambda_{1} x_{1}+\lambda_{2} x_{2}+\cdots+\lambda_{n} x_{n}\right\|^{2}=\lambda_{1}\left\|x_{1}\right\|^{2}+\lambda_{2}\left\|x_{1}\right\|^{2}+\cdots+\lambda_{n}\left\|x_{n}\right\|^{2}-\sum_{1 \leqslant i \leqslant j \leqslant n} \lambda_{i} \lambda_{j}\left\|x_{i}-x_{j}\right\|^{2} .
$$

Lemma 2.2 ([14]). Let $\left\{\alpha_{n}\right\}$ be a sequence of real numbers such that there exists a subsequence $\left\{n_{i}\right\}$ of $\{n\}$ such that $\alpha_{n_{i}}<\alpha_{n_{i}+1}$ for all $i \in \mathbb{N}$. Then there exists a nondecreasing sequence $\left\{m_{k}\right\} \subseteq \mathbb{N}$ such that $m_{k} \rightarrow \infty$ and the following properties are satisfied for all (sufficiently large) numbers $k \in \mathbb{N}$ :

$$
\alpha_{m_{k}} \leqslant \alpha_{m_{k}+1} \text { and } \alpha_{k} \leqslant \alpha_{m_{k}+1} .
$$

In fact, $m_{k}=\max \left\{j \leqslant k: \alpha_{j}<\alpha_{j+1}\right\}$.

Lemma 2.3 ([29]). Let $\left\{\alpha_{n}\right\}$ be a sequence of nonnegative numbers satisfying the property:

$$
\alpha_{n+1} \leqslant\left(1-\gamma_{n}\right) \alpha_{n}+b_{n}+\gamma_{n} c_{n}, \quad n \in \mathbb{N},
$$

where $\left\{\gamma_{n}\right\},\left\{b_{n}\right\},\left\{c_{n}\right\}$ satisfy the restrictions:

(i) $\sum_{n=1}^{\infty} \gamma_{n}=\infty, \quad \lim _{n \rightarrow \infty} \gamma_{n}=0$;

(ii) $b_{n} \geqslant 0, \quad \sum_{n=1}^{\infty} b_{n}<\infty$;

(iii) $\lim \sup _{n \rightarrow \infty} c_{n} \leqslant 0$.

Then, $\lim _{n \rightarrow \infty} \alpha_{n}=0$.

Let $T: C \rightarrow E$ be a mapping. A point $p \in C$ is said to be an asymptotic fixed point of $T$ if there exists a sequence $\left\{x_{n}\right\}$ of $C$ such that $x_{n} \rightarrow p$ and $x_{n}-T x_{n} \rightarrow 0$; see [16]. The set of all asymptotic fixed points of $\mathrm{T}$ is denoted by $\mathrm{F}(\hat{\mathrm{T}})$.

Lemma 2.4 ([3]). If $\mathrm{T}: \mathrm{C} \rightarrow \mathrm{E}$ is a firmly nonexpansive-like mapping, then $\mathrm{F}(\mathrm{T})$ is a closed convex subset of $\mathrm{E}$ and $\mathrm{F}(\hat{\mathrm{T}})=\mathrm{F}(\mathrm{T})$.

Lemma 2.5 ([1]). Let $\mathrm{C}$ be a nonempty close convex subset of a real Hilbert space $\mathrm{H}$. Let $\mathrm{k} \in(-\infty, 1)$ and $\mathrm{T}$ a $\mathrm{k}$-demimetric mapping of $\mathrm{C}$ into $\mathrm{H}$ such that $\mathrm{F}(\mathrm{T})$ is nonempty. Let $\mathrm{l}$ be a real number with $\mathrm{l} \in(0, \infty)$ and define $\mathrm{S}=(1-\mathrm{l}) \mathrm{I}+\mathrm{lT}$. Then there holds that

(1) $\mathrm{F}(\mathrm{S})=\mathrm{F}(\mathrm{T})$ if $\mathrm{l} \neq 0$; 
(2) $\mathrm{S}$ is a quasi-nonexpansive mapping for $\mathrm{l} \in(0,1-\mathrm{k}]$;

(3) $\mathrm{F}(\mathrm{T})$ is a closed convex subset of $\mathrm{H}$.

Lemma 2.6 ([15]). Let $\mathrm{C}$ be a nonempty closed convex subset of a real Hilbert space $\mathrm{H}$. Given $\mathrm{x} \in \mathrm{H}$ and $z \in \mathrm{C}$, then $z=\mathrm{P}_{\mathrm{C}} \mathrm{x}$ if and only if there holds the relation

$$
\langle x-z, y-z\rangle \leqslant 0, \quad \forall y \in C,
$$

where $\mathrm{P}_{\mathrm{C}}$ is the metric projection of $\mathrm{H}$ onto $\mathrm{C}$.

\section{Main results}

We also need the following lemmas which are fundamental for our theorems.

Lemma 3.1. Let $\mathrm{E}$ be a smooth, strictly convex, and reflexive Banach space and let $\mathrm{J}$ be the duality mapping on $\mathrm{E}$. Let $\mathrm{H}$ be a Hilbert space and let $\mathrm{C}$ be a nonempty closed convex subset of $\mathrm{H}$. Let $\mathrm{A}: \mathrm{H} \rightarrow 2^{\mathrm{H}}$ be a maximal monotone operator such that $\operatorname{dom}(\mathrm{A}) \subset \mathrm{C}$ and let $\mathrm{J}_{\lambda}$ be the resolvent of $\mathrm{A}$ for $\lambda>0$. Let $\mathrm{T}: \mathrm{E} \rightarrow \mathrm{E}$ be a firmly nonexpansive-like mapping. Let $\mathrm{S}: \mathrm{H} \rightarrow \mathrm{E}$ be a bounded linear operator such that $\mathrm{S} \neq 0$ and let $\mathrm{S}^{*}$ be the adjoint operator of S. Suppose that $A^{-1} 0 \bigcap S^{-1} \mathrm{~F}(\mathrm{~T}) \neq \emptyset$. Then it holds, for all $\lambda, \mathrm{r}>0$, that

$$
\mathrm{F}\left(\mathrm{J}_{\lambda}\left(\mathrm{I}-\mathrm{rS} \mathrm{S}^{*} \mathrm{~J}(\mathrm{I}-\mathrm{T}) \mathrm{S}\right)\right)=\mathrm{A}^{-1} 0 \bigcap \mathrm{S}^{-1} \mathrm{~F}(\mathrm{~T}) \text {. }
$$

Proof. We first prove that

$$
\mathrm{F}\left(\mathrm{J}_{\lambda}\left(\mathrm{I}-\mathrm{r} \mathrm{S}^{*} \mathrm{~J}(\mathrm{I}-\mathrm{T}) \mathrm{S}\right)\right) \subseteq A^{-1} 0 \bigcap \mathrm{S}^{-1} \mathrm{~F}(\mathrm{~T}) .
$$

Suppose that $z \in F\left(J_{\lambda}\left(I-r S^{*} J(I-T) S\right)\right)$ and $z_{0} \in A^{-1} 0 \bigcap S^{-1} F(T)$. Then we have

$$
z=\mathrm{J}_{\lambda}\left(\mathrm{I}-\mathrm{r} S^{*} \mathrm{~J}(\mathrm{I}-\mathrm{T}) \mathrm{S}\right) z \Leftrightarrow\left(\mathrm{I}-\mathrm{r} \mathrm{S}^{*} \mathrm{~J}(\mathrm{I}-\mathrm{T}) \mathrm{S}\right) z \in(\mathrm{I}+\lambda \mathrm{A}) z \Leftrightarrow-\frac{\mathrm{r}}{\lambda} \mathrm{S}^{*} \mathrm{~J}(\mathrm{I}-\mathrm{T}) S z \in A z .
$$

Since $A$ is monotone and $0 \in A z_{0}$, we see that

$$
\left\langle z-z_{0}, S^{*} J(I-T) S z\right\rangle \leqslant 0 .
$$

And hence

$$
\left\langle\mathrm{S} z-\mathrm{S} z_{0}, \mathrm{~J}(\mathrm{I}-\mathrm{T}) \mathrm{S} z\right\rangle \leqslant 0 .
$$

On the other hand, since $T$ is a firmly nonexpansive-like mapping and $S z_{0} \in F(T)$, we have that

$$
\left\langle\mathrm{TS} z-\mathrm{S} z_{0}, \mathrm{~J}(\mathrm{I}-\mathrm{T}) \mathrm{S} z\right\rangle \geqslant 0 .
$$

Adding up (3.1) and (3.2), we find that

$$
\|S z-\mathrm{TS} z\|^{2}=\langle\mathrm{S} z-\mathrm{TS} z, \mathrm{~J}(\mathrm{I}-\mathrm{T}) \mathrm{S} z\rangle \leqslant 0 .
$$

Therefore, $S z=T S z$. That is, $z \in S^{-1} \mathrm{~F}(\mathrm{~T})$ and also $S z-\mathrm{TS} z=0$ implying $\left.\mathrm{S}^{*} \mathrm{~J}(\mathrm{I}-\mathrm{T}) \mathrm{S}\right) z=0$. This reduces the fixed point equation $z=\mathrm{J}_{\lambda}\left(\mathrm{I}-\mathrm{rS} \mathrm{S}^{*} \mathrm{~J}(\mathrm{I}-\mathrm{T}) \mathrm{S}\right) z$ to the fixed point equation $z=\mathrm{J}_{\lambda} z$ that is equivalent to $z \in A^{-1} 0$. Consequently, $z \in A^{-1} 0 \bigcap S^{-1} \mathrm{~F}(\mathrm{~T})$.

We now prove

$$
A^{-1} 0 \bigcap S^{-1} F(T) \subseteq F\left(J_{\lambda}\left(I-r S^{*} J(I-T) S\right)\right) .
$$

Since $z_{0} \in A^{-1} 0 \bigcap S^{-1} F(T)$, we have that $z_{0} \in A^{-1} 0$ and $z_{0} \in S^{-1} F(T)$. It follows that $z_{0}=\mathrm{J}_{\lambda} z_{0}$ and $S z_{0}=\mathrm{TS}_{0}$. Hence, we have

$$
z_{0}=\mathrm{J}_{\lambda}\left(\mathrm{I}-\mathrm{rS} \mathrm{J}^{*}(\mathrm{I}-\mathrm{T}) \mathrm{S}\right) z_{0}
$$

which implies $A^{-1} 0 \bigcap S^{-1} \mathrm{~F}(\mathrm{~T}) \subseteq \mathrm{F}\left(\mathrm{J}_{\lambda}\left(\mathrm{I}-\mathrm{r} \mathrm{S}^{*} \mathrm{~J}(\mathrm{I}-\mathrm{T}) S\right)\right)$. This completes the proof.

Lemma 3.2. In the setting of Lemma 3.1, if $r \in\left(0, \frac{2}{\|S\|^{2}}\right]$, then the mapping $\mathrm{J}_{\lambda}\left(\mathrm{I}-\mathrm{rS} \mathrm{S}^{*} \mathrm{~J}(\mathrm{I}-\mathrm{T}) \mathrm{S}\right)$ is quasinonexpansive on $\mathrm{H}$. 
Proof. Since the resolvent $J_{\lambda}$ is nonexpansive, we see that, for $u \in A^{-1} 0 \bigcap S^{-1} F(T)$,

$$
\begin{aligned}
\left\|J_{\lambda}\left(\mathrm{I}-\mathrm{r} S^{*} J(\mathrm{I}-\mathrm{T}) \mathrm{S}\right) z-\mathrm{u}\right\|^{2} & \leqslant\left\|z-r S^{*} J(S z-\mathrm{TS} z)-\mathrm{u}\right\|^{2} \\
& =\|z-u\|^{2}+\left\|r S^{*} J(S z-\mathrm{TS} z)\right\|^{2}-2 r\left\langle z-u, S^{*} J(S z-\mathrm{TS} z)\right\rangle \\
& \leqslant\|z-u\|^{2}+r^{2}\|S\|^{2}\|S z-\mathrm{TS} z\|^{2}-2 r\langle S z-\mathrm{TS} z+\mathrm{TS} z-\mathrm{Su}, \mathrm{J}(\mathrm{S} z-\mathrm{TS} z)\rangle \\
& \leqslant\|z-u\|^{2}+r^{2}\|S\|^{2}\|S z-\mathrm{TS} z\|^{2}-2 r\|S z-\mathrm{TS} z\|^{2}-2 r\langle\mathrm{TS} z-\mathrm{Su}, \mathrm{J}(\mathrm{S} z-\mathrm{TS} z)\rangle \\
& \leqslant\|z-u\|^{2}+r^{2}\|S\|^{2}\|S z-\mathrm{TS} z\|^{2}-2 r\|S z-\mathrm{TS} z\|^{2} \\
& =\|z-u\|^{2}-r\left(2-r\|S\|^{2}\right)\|S z-\mathrm{TS} z\|^{2} \\
& \leqslant\|z-u\|^{2} .
\end{aligned}
$$

From Lemma 3.1, we see $u \in F\left(J_{\lambda}\left(I-r S^{*} J(I-T) S\right)\right)$. Thus we have the desired result.

Lemma 3.3. Let $\mathrm{H}$ be a Hilbert space and $\mathrm{C}$ be a nonempty convex subset of $\mathrm{H}$. Assume that $\left\{\mathrm{T}_{i}\right\}_{i=1}^{\infty}: \mathrm{C} \rightarrow \mathrm{H}$ be an infinite family of $\mathrm{k}_{i}$-demimetric mappings with $\sup \left\{\mathrm{k}_{i}: i \in \mathbb{N}\right\}<1$ such that $\bigcap_{i=1}^{\infty} \mathrm{F}\left(\mathrm{T}_{i}\right) \neq \emptyset$. Assume that $\left\{\eta_{i}\right\}_{i=1}^{\infty}$ is a positive sequence such that $\sum_{i=1}^{\infty} \eta_{i}=1$. Then $\sum_{i=1}^{\infty} \eta_{i} T_{i}: C \rightarrow H$ is a k-demimetric mapping with $k=\sup \left\{k_{i}: i \in \mathbb{N}\right\}$ and $F\left(\sum_{i=1}^{\infty} \eta_{i} T_{i}\right)=\bigcap_{i=1}^{\infty} F\left(T_{i}\right)$.

Proof. Let

$$
\mathrm{G}_{\mathrm{n}} \mathrm{x}=\eta_{1} \mathrm{~T}_{1} x+\eta_{2} \mathrm{~T}_{2} x+\cdots+\eta_{n} \mathrm{~T}_{\mathrm{n}} x
$$

and $\sum_{i=1}^{n} \eta_{i}=1$. Then, $G_{n}: C \rightarrow H$ is a $k$-demimetric mapping with $k=\max \left\{k_{i}: 1 \leqslant i \leqslant n\right\}$. Indeed, we can firstly see the case of $n=2$.

$$
\begin{aligned}
\left\langle\left(I-G_{2}\right) x, x-q\right\rangle & =\left\langle\eta_{1}\left(I-T_{1}\right) x+\eta_{2}\left(I-T_{2}\right) x, x-q\right\rangle \\
& =\eta_{1}\left\langle x-T_{1} x, x-q\right\rangle+\eta_{2}\left\langle x-T_{2} x, x-q\right\rangle \\
& \geqslant \eta_{1} \frac{1-k_{1}}{2}\left\|x-T_{1} x\right\|^{2}+\eta_{2} \frac{1-k_{2}}{2}\left\|x-T_{2} x\right\|^{2} \\
& \geqslant \frac{1-k}{2}\left(\eta_{1}\left\|x-T_{1} x\right\|^{2}+\eta_{2}\left\|x-T_{2} x\right\|^{2}\right) \\
& \geqslant \frac{1-k}{2}\left\|x-G_{2} x\right\|^{2}
\end{aligned}
$$

where $\mathrm{q} \in \bigcap_{i=1}^{\infty} F\left(T_{i}\right)$. This shows that $G_{2}: C \rightarrow H$ is a k-demimetric mapping with $k=\max \left\{k_{i}: i=1,2\right\}$. By the same way, our proof method easily carries over to the general finite case.

Next, we prove the infinite case. From the definition of demimetric mapping, we know

$$
\left\langle x-\mathrm{T}_{i} x, x-\mathrm{q}\right\rangle \geqslant \frac{1-\mathrm{k}_{\mathrm{i}}}{2}\left\|x-\mathrm{T}_{i} x\right\|^{2} .
$$

Hence, we can get

$$
\left\|\mathrm{T}_{i} x\right\| \leqslant\left\|x-\mathrm{T}_{i} x\right\|+\|x\| \leqslant \frac{2}{1-\mathrm{k}_{i}}\|x-q\|+\|x\| \leqslant \frac{2}{1-k}\|x-q\|+\|x\| .
$$

Then, we deduce $\sum_{i=1}^{\infty} \eta_{i} T_{i}$ strongly converges. Letting

$$
\mathrm{T} x=\sum_{i=1}^{\infty} \eta_{i} \mathrm{~T}_{i} x
$$

we have

$$
T x=\sum_{i=1}^{\infty} \eta_{i} T_{i} x=\lim _{n \rightarrow \infty} \sum_{i=1}^{n} \eta_{i} T_{i} x=\lim _{n \rightarrow \infty} \frac{1}{\sum_{i=1}^{n} \eta_{i}} \sum_{i=1}^{n} \eta_{i} T_{i} x
$$


Therefore, we get

$$
\begin{aligned}
\langle x-T x, x-q\rangle & =\lim _{n \rightarrow \infty}\left\langle\left(I-\frac{1}{\sum_{i=1}^{n} \eta_{i}} \sum_{i=1}^{n} \eta_{i} T_{i}\right) x, x-q\right\rangle \\
& =\lim _{n \rightarrow \infty} \frac{1}{\sum_{i=1}^{n} \eta_{i}} \sum_{i=1}^{n} \eta_{i}\left\langle\left(I-T_{i}\right) x, x-q\right\rangle \\
& \geqslant \lim _{n \rightarrow \infty} \frac{1}{\sum_{i=1}^{n} \eta_{i}} \sum_{i=1}^{n} \eta_{i} \frac{1-k_{i}}{2}\left\|x-T_{i} x\right\|^{2} \\
& \geqslant \lim _{n \rightarrow \infty} \frac{1}{\sum_{i=1}^{n} \eta_{i}} \sum_{i=1}^{n} \eta_{i} \frac{1-k}{2}\left\|x-T_{i} x\right\|^{2} \\
& \geqslant \frac{1-k}{2} \lim _{n \rightarrow \infty}\left\|\left(I-\frac{1}{\sum_{i=1}^{n} \eta_{i}} \sum_{i=1}^{n} \eta_{i} T_{i}\right) x\right\|^{2} \\
& =\frac{1-k}{2}\|x-T x\|^{2} .
\end{aligned}
$$

So, we deduce that $\mathrm{T}$ is $\mathrm{k}$-demimetric.

Finally, we show $F\left(\sum_{i=1}^{\infty} \eta_{i} T_{i}\right)=\bigcap_{i=1}^{\infty} F\left(T_{i}\right)$. Suppose that $x=\sum_{i=1}^{\infty} \eta_{i} T_{i} x$, it is sufficient to show that $x \in \bigcap_{i=1}^{\infty} F\left(T_{i}\right)$. Indeed, for $p \in \bigcap_{i=1}^{\infty} F\left(T_{i}\right)$, we have

$$
\begin{aligned}
\|x-p\|^{2}=\langle x-p, x-p\rangle & =\left\langle\sum_{i=1}^{\infty} \eta_{i} T_{i} x-p, x-p\right\rangle \\
& =\sum_{i=1}^{\infty} \eta_{i}\left\langle T_{i} x-p, x-p\right\rangle \\
& =\sum_{i=1}^{\infty} \eta_{i}\left(\langle x-p, x-p\rangle+\left\langle T_{i} x-x, x-p\right\rangle\right) \\
& \leqslant\|x-p\|^{2}-\sum_{i=1}^{\infty} \eta_{i} \frac{1-k_{i}}{2}\left\|x-T_{i} x\right\|^{2} \\
& \leqslant\|x-p\|^{2}-\frac{1-k}{2} \sum_{i=1}^{\infty} \eta_{i}\left\|x-T_{i} x\right\|^{2},
\end{aligned}
$$

where $k=\sup \left\{k_{i}: i \in \mathbb{N}\right\}$. Hence, we deduce $x=T_{i} x$ for each $i \in \mathbb{N}$. This means that $x \in \bigcap_{i=1}^{\infty} F\left(T_{i}\right)$.

Theorem 3.4. Let $\mathrm{E}$ be a smooth, strictly convex, and reflexive Banach space and let $\mathrm{J}$ be the duality mapping on $\mathrm{E}$. Let $\mathrm{H}$ be a Hilbert space and let $\mathrm{C}$ be a nonempty closed convex subset of $\mathrm{H}$. Let $\mathrm{A}$ be a maximal monotone operator of $\mathrm{H}$ into $2^{\mathrm{H}}$ such that $\operatorname{dom}(\mathrm{A}) \subset \mathrm{C}$. Let $\mathrm{J}_{\lambda}$ be the resolvent of $\mathrm{A}$ for $\lambda>0$ and let $\mathrm{T}$ be a firmly nonexpansive-like mapping on $\mathrm{E}$. Let $\mathrm{S}: \mathrm{H} \rightarrow \mathrm{E}$ be a bounded linear operator such that $\mathrm{S} \neq 0$ and let $\mathrm{S}^{*}$ be the adjoint operator of $\mathrm{S}$. Let $\left\{\mathrm{U}_{i}\right\}_{i=1}^{\infty}: \mathrm{C} \rightarrow \mathrm{H}$ be an infinite family of $\mathrm{k}_{i}$-demimetric and demiclosed mappings. Assume $\Gamma:=\bigcap_{i=1}^{\infty} \mathrm{F}\left(\mathrm{U}_{i}\right) \cap A^{-1} 0 \bigcap S^{-1} \mathrm{~F}(\mathrm{~T})$ is nonempty and the element $\mathrm{x}_{0} \in \mathrm{C}$ is fixed. Let $\left\{\mathrm{x}_{\mathrm{n}}\right\}$ be a sequence generated by

$$
\left\{\begin{array}{l}
x_{1} \quad \text { chosen arbitrarily, } \\
z_{n}=J_{\lambda_{n}}\left(x_{n}-\delta_{n} S^{*} J\left(S x_{n}-T S x_{n}\right)\right), \\
y_{n}=\left(1-\sigma_{n}\right) z_{n}+\sigma_{n} \sum_{i=1}^{\infty} \eta_{i} U_{i} z_{n}, \\
x_{n+1}=P_{C}\left(\alpha_{n} x_{0}+\beta_{n} y_{n}+\gamma_{n} z_{n}\right), \quad n \in \mathbb{N},
\end{array}\right.
$$

where $\left\{\alpha_{n}\right\},\left\{\beta_{n}\right\},\left\{\gamma_{n}\right\},\left\{\eta_{i}\right\} \subset(0,1)$ and $\left\{\lambda_{n}\right\},\left\{\delta_{n}\right\},\left\{\sigma_{n}\right\} \subset(0,+\infty)$ satisfy the following conditions: 
(i) $\lim _{n \rightarrow \infty} \alpha_{n}=0$ and $\sum_{n=1}^{\infty} \alpha_{n}=\infty$;

(ii) $0<\liminf _{n \rightarrow \infty} \beta_{n} \leqslant \limsup _{n \rightarrow \infty} \beta_{n}<1$ and $\alpha_{n}+\beta_{n}+\gamma_{n}=1$;

(iii) $\sum_{i=1}^{\infty} \eta_{i}=1$;

(iv) $0<a \leqslant \sigma_{n} \leqslant b<1-k$ and $k=\sup \left\{k_{i}: i \in \mathbb{N}\right\}<1$;

(v) $0<\mathrm{c} \leqslant \delta_{\mathrm{n}} \leqslant \mathrm{d}<\frac{2}{\|\mathrm{~S}\|^{2}}$ and $0<\liminf _{\mathrm{n} \rightarrow \infty} \lambda_{\mathrm{n}} \leqslant \limsup _{\mathrm{n} \rightarrow \infty} \lambda_{\mathrm{n}}<\infty$.

Then the sequence $\left\{x_{n}\right\}$ generated by (3.3) converges strongly to a point $z_{0} \in \Gamma$, where $z_{0}=\mathrm{P}_{\Gamma} x_{0}$.

Proof. Set $V=\sum_{i=1}^{\infty} \eta_{i} U_{i}$ and $W_{n}=\left(1-\sigma_{n}\right) I+\sigma_{n} V$. Since $W_{n}$ is quasi-nonexpansive with $F\left(W_{n}\right)=$ $F(V)=\bigcap_{i=1}^{\infty} F\left(U_{i}\right)$ due to Lemma 2.5 and Lemma 3.3, we have by Lemma 3.2, for any $u \in \Gamma$ that

$$
\begin{aligned}
\left\|x_{n+1}-u\right\| & \leqslant\left\|\alpha_{n}\left(x_{0}-u\right)+\beta_{n}\left(y_{n}-u\right)+\gamma_{n}\left(z_{n}-u\right)\right\| \\
& \leqslant \alpha_{n}\left\|x_{0}-u\right\|+\beta_{n}\left\|y_{n}-u\right\|+\gamma_{n}\left\|z_{n}-u\right\| \\
& \leqslant \alpha_{n}\left\|x_{0}-u\right\|+\beta_{n}\left\|W_{n} z_{n}-u\right\|+\gamma_{n}\left\|z_{n}-u\right\| \\
& \leqslant \alpha_{n}\left\|x_{0}-u\right\|+\beta_{n}\left\|z_{n}-u\right\|+\gamma_{n}\left\|z_{n}-u\right\| \\
& \leqslant \alpha_{n}\left\|x_{0}-u\right\|+\left(1-\alpha_{n}\right)\left\|J_{\lambda_{n}}\left(x_{n}-\delta_{n} S^{*} J\left(S x_{n}-T S x_{n}\right)\right)-u\right\| \\
& \leqslant \alpha_{n}\left\|x_{0}-u\right\|+\left(1-\alpha_{n}\right)\left\|x_{n}-u\right\| \\
& \leqslant \max \left\{\left\|x_{0}-u\right\|,\left\|x_{n}-u\right\|\right\} .
\end{aligned}
$$

By induction, we obtain

$$
\left\|x_{n}-u\right\| \leqslant \max \left\{\left\|x_{0}-u\right\|,\left\|x_{1}-u\right\|\right\}, \quad \forall n \in \mathbb{N},
$$

which gives that the sequence $\left\{x_{n}\right\}$ is bounded, so are $\left\{y_{n}\right\}$ and $\left\{z_{n}\right\}$.

In terms of Lemma 2.1 and Lemma 2.5, we have

$$
\begin{aligned}
\left\|x_{n+1}-u\right\|^{2} & =\left\|\alpha_{n}\left(x_{0}-u\right)+\beta_{n}\left(y_{n}-u\right)+\gamma_{n}\left(z_{n}-u\right)\right\|^{2} \\
& \leqslant \alpha_{n}\left\|x_{0}-u\right\|^{2}+\beta_{n}\left\|y_{n}-u\right\|^{2}+\gamma_{n}\left\|z_{n}-u\right\|^{2}-\beta_{n} \gamma_{n}\left\|y_{n}-z_{n}\right\|^{2} \\
& \leqslant \alpha_{n}\left\|x_{0}-u\right\|^{2}+\beta_{n}\left\|z_{n}-u\right\|^{2}+\gamma_{n}\left\|z_{n}-u\right\|^{2}-\beta_{n} \gamma_{n}\left\|y_{n}-z_{n}\right\|^{2} \\
& \leqslant \alpha_{n}\left\|x_{0}-u\right\|^{2}+\left(1-\alpha_{n}\right)\left\|z_{n}-u\right\|^{2}-\beta_{n} \gamma_{n}\left\|y_{n}-z_{n}\right\|^{2} .
\end{aligned}
$$

Now put $J_{\lambda_{n}}=\frac{1}{2}\left(I+K_{n}\right)$ for all $n \in \mathbb{N}$. Since $J_{\lambda_{n}}$ is firmly nonexpansive, then we know that $K_{n}$ is nonexpansive and $F\left(J_{\lambda_{n}}\right)=F\left(K_{n}\right)$ for all $n \in \mathbb{N}$. Therefore, we get

$$
\begin{aligned}
\left\|z_{n}-u\right\|^{2}= & \left\|J_{\lambda_{n}}\left(x_{n}-\delta_{n} S^{*} J\left(S x_{n}-T S x_{n}\right)\right)-u\right\|^{2} \\
= & \frac{1}{2}\left\|\left(x_{n}-\delta_{n} S^{*} J\left(S x_{n}-T S x_{n}\right)\right)-u\right\|^{2}+\frac{1}{2}\left\|K_{n}\left(x_{n}-\delta_{n} S^{*} J\left(S x_{n}-T S x_{n}\right)\right)-u\right\|^{2} \\
& -\frac{1}{4}\left\|K_{n}\left(x_{n}-\delta_{n} S^{*} J\left(S x_{n}-T S x_{n}\right)\right)-\left(x_{n}-\delta_{n} S^{*} J\left(S x_{n}-T S x_{n}\right)\right)\right\|^{2} \\
\leqslant & \left\|x_{n}-\delta_{n} S^{*} J\left(S x_{n}-T S x_{n}\right)-u\right\|^{2} \\
& -\frac{1}{4}\left\|K_{n}\left(x_{n}-\delta_{n} S^{*} J\left(S x_{n}-T S x_{n}\right)\right)-\left(x_{n}-\delta_{n} S^{*} J\left(S x_{n}-T S x_{n}\right)\right)\right\|^{2} \\
\leqslant & \left\|x_{n}-u\right\|^{2}+\left\|\delta_{n} S^{*} J\left(S x_{n}-T S x_{n}\right)\right\|^{2}-2 \delta_{n}\left\langle x_{n}-u, S^{*} J\left(S x_{n}-T S x_{n}\right)\right\rangle \\
& -\frac{1}{4}\left\|K_{n}\left(x_{n}-\delta_{n} S^{*} J\left(S x_{n}-T S x_{n}\right)\right)-\left(x_{n}-\delta_{n} S^{*} J\left(S x_{n}-T S x_{n}\right)\right)\right\|^{2} \\
\leqslant & \left\|x_{n}-u\right\|^{2}+\left\|\delta_{n} S^{*} J\left(S x_{n}-T S x_{n}\right)\right\|^{2}-2 \delta_{n}\left\langle S x_{n}-T S x_{n}+T S x_{n}-S u, J\left(S x_{n}-T S x_{n}\right)\right\rangle \\
& -\frac{1}{4}\left\|K_{n}\left(x_{n}-\delta_{n} S^{*} J\left(S x_{n}-T S x_{n}\right)\right)-\left(x_{n}-\delta_{n} S^{*} J\left(S x_{n}-T S x_{n}\right)\right)\right\|^{2} \\
\leqslant & \left\|x_{n}-u\right\|^{2}+\delta_{n}^{2}\|S\|^{2}\left\|S x_{n}-T S x_{n}\right\|^{2}-2 \delta_{n}\left\|S x_{n}-T S x_{n}\right\|^{2}-2 \delta_{n}\left\langle T S x_{n}-S u, J\left(S x_{n}-T S x_{n}\right)\right\rangle
\end{aligned}
$$




$$
\begin{aligned}
& -\frac{1}{4}\left\|K_{n}\left(x_{n}-\delta_{n} S^{*} J\left(S x_{n}-T S x_{n}\right)\right)-\left(x_{n}-\delta_{n} S^{*} J\left(S x_{n}-T S x_{n}\right)\right)\right\|^{2} \\
\leqslant & \left\|x_{n}-u\right\|^{2}+\delta_{n}^{2}\|S\|^{2}\left\|S x_{n}-T S x_{n}\right\|^{2}-2 \delta_{n}\left\|S x_{n}-T S x_{n}\right\|^{2} \\
& -\frac{1}{4}\left\|K_{n}\left(x_{n}-\delta_{n} S^{*} J\left(S x_{n}-T S x_{n}\right)\right)-\left(x_{n}-\delta_{n} S^{*} J\left(S x_{n}-T S x_{n}\right)\right)\right\|^{2} \\
= & \left\|x_{n}-u\right\|^{2}-\delta_{n}\left(2-\delta_{n}\|S\|^{2}\right)\left\|S x_{n}-T S x_{n}\right\|^{2} \\
& -\frac{1}{4}\left\|K_{n}\left(x_{n}-\delta_{n} S^{*} J\left(S x_{n}-T S x_{n}\right)\right)-\left(x_{n}-\delta_{n} S^{*} J\left(S x_{n}-T S x_{n}\right)\right)\right\|^{2} .
\end{aligned}
$$

It follows from (3.4) and (3.5) that

$$
\begin{aligned}
\left\|x_{n+1}-u\right\|^{2} \leqslant & \alpha_{n}\left\|x_{0}-u\right\|^{2}+\left\|x_{n}-u\right\|^{2}-\delta_{n}\left(2-\delta_{n}\|S\|^{2}\right)\left\|S x_{n}-T S x_{n}\right\|^{2} \\
& -\frac{1}{4}\left\|K_{n}\left(x_{n}-\delta_{n} S^{*} J\left(S x_{n}-T S x_{n}\right)\right)-\left(x_{n}-\delta_{n} S^{*} J\left(S x_{n}-T S x_{n}\right)\right)\right\|^{2} \\
& -\beta_{n} \gamma_{n}\left\|y_{n}-z_{n}\right\|^{2},
\end{aligned}
$$

which implies that

$$
\begin{aligned}
\delta_{n}(2 & \left.-\delta_{n}\|S\|^{2}\right)\left\|S x_{n}-T S x_{n}\right\|^{2}+\beta_{n} \gamma_{n}\left\|y_{n}-z_{n}\right\|^{2} \\
& +\frac{1}{4}\left\|K_{n}\left(x_{n}-\delta_{n} S^{*} J\left(S x_{n}-T S x_{n}\right)\right)-\left(x_{n}-\delta_{n} S^{*} J\left(S x_{n}-T S x_{n}\right)\right)\right\|^{2} \\
\leqslant & \alpha_{n}\left\|x_{0}-u\right\|^{2}+\left\|x_{n}-u\right\|^{2}-\left\|x_{n+1}-u\right\|^{2} .
\end{aligned}
$$

Case 1. Assume there exists some integer $m>0$ such that $\left\{\left\|x_{n}-z_{0}\right\|\right\}$ is decreasing for all $n \geqslant m$. In this case, we know that $\lim _{n \rightarrow \infty}\left\|x_{n}-u\right\|$ exists. From (3.6), and conditions (i), (ii), and (v), we deduce

$$
\begin{aligned}
& \lim _{n \rightarrow \infty}\left\|y_{n}-z_{n}\right\|=0, \\
& \lim _{n \rightarrow \infty}\left\|K_{n}\left(x_{n}-\delta_{n} S^{*} J\left(S x_{n}-T S x_{n}\right)\right)-\left(x_{n}-\delta_{n} S^{*} J\left(S x_{n}-T S x_{n}\right)\right)\right\|=0,
\end{aligned}
$$

and

$$
\lim _{n \rightarrow \infty}\left\|S x_{n}-T S x_{n}\right\|=0
$$

Notice that

$$
\begin{aligned}
\left\|z_{n}-x_{n}\right\|= & \left\|J_{\lambda_{n}}\left(x_{n}-\delta_{n} S^{*} J\left(S x_{n}-T S x_{n}\right)\right)-x_{n}\right\| \\
= & \left\|\frac{1}{2}\left(x_{n}-\delta_{n} S^{*} J\left(S x_{n}-T S x_{n}\right)\right)+\frac{1}{2} K_{n}\left(x_{n}-\delta_{n} S^{*} J\left(S x_{n}-T S x_{n}\right)\right)-x_{n}\right\| \\
\leqslant & \frac{1}{2}\left\|\delta_{n} S^{*} J\left(S x_{n}-T S x_{n}\right)\right\|+\frac{1}{2}\left\|K_{n}\left(x_{n}-\delta_{n} S^{*} J\left(S x_{n}-T S x_{n}\right)\right)-x_{n}\right\| \\
\leqslant & \frac{1}{2}\left\|\delta_{n} S^{*} J\left(S x_{n}-T S x_{n}\right)\right\|+\frac{1}{2}\left\|\left(x_{n}-\delta_{n} S^{*} J\left(S x_{n}-T S x_{n}\right)\right)-x_{n}\right\| \\
& +\frac{1}{2}\left\|K_{n}\left(x_{n}-\delta_{n} S^{*} J\left(S x_{n}-T S x_{n}\right)\right)-\left(x_{n}-\delta_{n} S^{*} J\left(S x_{n}-T S x_{n}\right)\right)\right\| \\
\leqslant & \delta_{n}\|S\|\left\|S x_{n}-T S x_{n}\right\|+\frac{1}{2}\left\|K_{n}\left(x_{n}-\delta_{n} S^{*} J\left(S x_{n}-T S x_{n}\right)\right)-\left(x_{n}-\delta_{n} S^{*} J\left(S x_{n}-T S x_{n}\right)\right)\right\|
\end{aligned}
$$

This together with (3.8) and (3.9) implies

$$
\lim _{n \rightarrow \infty}\left\|z_{n}-x_{n}\right\|=0 .
$$

Observe that

$$
\left\|J_{\lambda_{n}} x_{n}-x_{n}\right\| \leqslant\left\|J_{\lambda_{n}} x_{n}-z_{n}\right\|+\left\|z_{n}-x_{n}\right\|
$$




$$
\begin{aligned}
& \leqslant\left\|\delta_{n} S^{*} J\left(S x_{n}-T S x_{n}\right)\right\|+\left\|z_{n}-x_{n}\right\| \\
& \leqslant \delta_{n}\|S\|\left\|S x_{n}-T S x_{n}\right\|+\left\|z_{n}-x_{n}\right\| .
\end{aligned}
$$

It follows from (3.9) and (3.10) that

$$
\lim _{n \rightarrow \infty}\left\|J_{\lambda_{n}} x_{n}-x_{n}\right\|=0
$$

Taking into consideration that

$$
\left\|x_{n}-y_{n}\right\| \leqslant\left\|x_{n}-z_{n}\right\|+\left\|z_{n}-y_{n}\right\|,
$$

we deduce from (3.7) and (3.10) that

$$
\lim _{n \rightarrow \infty}\left\|x_{n}-y_{n}\right\|=0
$$

In view of (3.3), we get

$$
\left\|x_{n+1}-x_{n}\right\|=\left\|P_{C}\left(\alpha_{n} x_{0}+\beta_{n} y_{n}+\gamma_{n} z_{n}\right)-x_{n}\right\| \leqslant \alpha_{n}\left\|x_{0}-x_{n}\right\|+\beta_{n}\left\|y_{n}-x_{n}\right\|+\gamma_{n}\left\|z_{n}-x_{n}\right\| .
$$

This together with (3.10), (3.12), and condition (i) implies that

$$
\lim _{n \rightarrow \infty}\left\|x_{n+1}-x_{n}\right\|=0
$$

Since $\left\{x_{n}\right\}$ is bounded, there exists a subsequence $\left\{x_{n_{i}}\right\}$ of $\left\{x_{n}\right\}$ satisfying $x_{n_{i}} \rightarrow \tilde{x} \in C$. Without loss of generality, we may also assume

$$
\lim _{i \rightarrow \infty}\left\langle x_{0}-z_{0}, x_{n_{i}}-z_{0}\right\rangle=\limsup _{n \rightarrow \infty}\left\langle x_{0}-z_{0}, x_{n}-z_{0}\right\rangle .
$$

It is clear that

$$
\begin{aligned}
\left\langle z_{n}-z_{0}, z_{n}-y_{n}\right\rangle=\sigma_{n}\left\langle z_{n}-z_{0}, z_{n}-v z_{n}\right\rangle & =\sigma_{n} \sum_{i=1}^{\infty} \eta_{i}\left\langle z_{n}-z_{0}, z_{n}-u_{i} z_{n}\right\rangle \\
& \geqslant \sigma_{n} \sum_{i=1}^{\infty} \eta_{i} \frac{1-k_{i}}{2}\left\|z_{n}-u_{i} z_{n}\right\| \geqslant \sigma_{n} \frac{1-k}{2} \sum_{i=1}^{\infty} \eta_{i}\left\|z_{n}-u_{i} z_{n}\right\| .
\end{aligned}
$$

By (3.7) and condition (iv), we conclude that

$$
\lim _{n \rightarrow \infty}\left\|z_{n}-u_{i} z_{n}\right\|=0, \quad \forall i \in \mathbb{N} .
$$

Since $U_{i}$ is demiclosed for each $i \in \mathbb{N}$, noticing (3.10), we have $\tilde{x} \in \bigcap_{i=1}^{\infty} F\left(U_{i}\right)$.

Let us show that $\tilde{x} \in A^{-1} 0 \cap S^{-1} F(T)$. Since $S$ is bounded and linear, we see that $S x_{n_{i}} \rightarrow S \tilde{x}$ as $i \rightarrow \infty$. Noticing (3.9) and Lemma 2.4, we deduce $S \tilde{x} \in F(T)$; that is $\tilde{x}=S^{-1} F(T)$. Since $J_{\lambda_{n}}$ is the resolvent of $A$ for $\lambda_{n}>0$, then we obtain $\frac{x_{n}-J_{\lambda_{n}} x_{n}}{\lambda_{n}} \in A J_{\lambda_{n}} x_{n}$ for all $n \geqslant 1$. From the monotonicity of $A$, we see that

$$
0 \leqslant\left\langle u-J_{\lambda_{n_{i}}} x_{n_{i}}, \tilde{u}-\frac{x_{n_{i}}-J_{\lambda_{n_{i}}} x_{n_{i}}}{\lambda_{n_{i}}}\right\rangle, \quad \forall(u, \tilde{u}) \in A .
$$

Letting $i \rightarrow \infty$, we deduce from (3.11) that

$$
0 \leqslant\langle u-\tilde{x}, \tilde{u}-0\rangle, \quad \forall(u, \tilde{u}) \in A .
$$

Since $A$ is maximal monotone, we get $\tilde{x} \in A^{-1} 0$. Therefor, we have $\tilde{x} \in A^{-1} 0 \bigcap S^{-1} F(T)$. And hence it follows from (3.13) that

$$
\limsup _{n \rightarrow \infty}\left\langle x_{0}-z_{0}, x_{n}-z_{0}\right\rangle=\lim _{i \rightarrow \infty}\left\langle x_{0}-z_{0}, x_{n_{i}}-z_{0}\right\rangle=\left\langle x_{0}-z_{0}, \tilde{x}-z_{0}\right\rangle=\left\langle x_{0}-P_{\Gamma} x_{0}, \tilde{x}-P_{\Gamma} x_{0}\right\rangle \leqslant 0 .
$$


Setting $h_{n}=\alpha_{n} x_{0}+\beta_{n} y_{n}+\gamma_{n} z_{n}$ for all $n \in \mathbb{N}$, we have from (3.3) that $x_{n+1}=P_{C} h_{n}$. It follows from Lemma 3.2 and Lemma 2.6 that

$$
\begin{aligned}
\left\|x_{n+1}-z_{0}\right\|^{2} & =\left\langle P_{C} h_{n}-h_{n}, P_{C} h_{n}-z_{0}\right\rangle+\left\langle h_{n}-z_{0}, P_{C} h_{n}-z_{0}\right\rangle \\
& \leqslant\left\langle\alpha_{n} x_{0}+\beta_{n} y_{n}+\gamma_{n} z_{n}-z_{0}, x_{n+1}-z_{0}\right\rangle \\
& \leqslant\left\|\beta_{n}\left(y_{n}-z_{0}\right)+\gamma_{n}\left(z_{n}-z_{0}\right)\right\|\left\|x_{n+1}-z_{0}\right\|+\alpha_{n}\left\langle x_{0}-z_{0}, x_{n+1}-z_{0}\right\rangle \\
& \leqslant \beta_{n}\left\|z_{n}-z_{0}\right\|\left\|x_{n+1}-z_{0}\right\|+\gamma_{n}\left\|z_{n}-z_{0}\right\|\left\|x_{n+1}-z_{0}\right\|+\alpha_{n}\left\langle x_{0}-z_{0}, x_{n+1}-z_{0}\right\rangle \\
& \leqslant\left(1-\alpha_{n}\right)\left\|x_{n}-z_{0}\right\|\left\|x_{n+1}-z_{0}\right\|+\alpha_{n}\left\langle x_{0}-z_{0}, x_{n+1}-z_{0}\right\rangle \\
& \leqslant\left(1-\alpha_{n}\right)\left\|x_{n}-z_{0}\right\|^{2}+\alpha_{n}\left\langle x_{0}-z_{0}, x_{n+1}-z_{0}\right\rangle .
\end{aligned}
$$

Applying Lemma 2.3 and (3.14) to (3.15), we deduce $x_{n} \rightarrow z_{0}$ as $n \rightarrow \infty$.

Case 2. Suppose that there exists $\left\{n_{i}\right\}$ of $\{n\}$ such that $\left\|x_{n_{i}}-z_{0}\right\|<\left\|x_{n_{i}+1}-z_{0}\right\|$ for all $i \in \mathbb{N}$. Then by Lemma 2.2, there exists a nondecreasing sequence $\left\{m_{j}\right\}$ in $\mathbb{N}$ such that

$$
\left\|x_{m_{j}}-z_{0}\right\| \leqslant\left\|x_{m_{j}+1}-z_{0}\right\| \quad \text { and } \quad\left\|x_{j}-z_{0}\right\| \leqslant\left\|x_{m_{j}+1}-z_{0}\right\| .
$$

We want to show that

$$
\limsup _{j \rightarrow \infty}\left\langle x_{0}-z_{0}, x_{m_{j}}-z_{0}\right\rangle \leqslant 0,
$$

where $z_{0}=P_{\Gamma} x_{0}$. Without loss of generality, there exists a subsequence $\left\{x_{m_{j_{k}}}\right\}$ of $\left\{x_{m_{j}}\right\}$ such that $x_{m_{j_{k}}} \rightarrow \omega$ for some $\omega \in C$ and

$$
\lim _{k \rightarrow \infty}\left\langle x_{0}-z_{0}, x_{m_{j_{k}}}-z_{0}\right\rangle=\limsup _{j \rightarrow \infty}\left\langle x_{0}-z_{0}, x_{m_{j}}-z_{0}\right\rangle .
$$

Following a similar argument as in the proof of Case 1, we have that

$$
\lim _{j \rightarrow \infty}\left\|z_{m_{j}}-x_{m_{j}}\right\|=0 \quad \text { and } \quad \lim _{j \rightarrow \infty}\left\|z_{\mathfrak{m}_{j}}-u_{i} z_{m_{j}}\right\|=0, \quad \forall i \in \mathbb{N} .
$$

By the assumption that $U_{i}$ is demiclosed for each $i \in \mathbb{N}$ and noticing (3.16), we deduce that $\omega \in$ $\bigcap_{i=1}^{\infty} F\left(U_{i}\right)$. Like in Case 1 , we can also obtain

$$
\omega \in A^{-1} 0 \bigcap S^{-1} F(T)
$$

and

$$
\lim _{j \rightarrow \infty}\left\|x_{m_{j}+1}-x_{m_{j}}\right\|=0
$$

Thus we obtain

$$
\limsup _{j \rightarrow \infty}\left\langle x_{0}-z_{0}, x_{m_{j}}-z_{0}\right\rangle=\lim _{k \rightarrow \infty}\left\langle x_{0}-z_{0}, x_{m_{j_{k}}}-z_{0}\right\rangle=\left\langle x_{0}-P_{\Gamma} x_{0}, \omega-P_{\Gamma} x_{0}\right\rangle \leqslant 0 .
$$

Letting $h_{m_{j}}=\alpha_{m_{j}} x_{0}+\beta_{m_{j}} y_{m_{j}}+\gamma_{m_{j}} z_{m_{j}}$ for all $j \in \mathbb{N}$, we deduce from (3.3) and Lemma 3.2 that

$$
\begin{aligned}
\left\|x_{m_{j}+1}-z_{0}\right\|^{2} & =\left\langle\mathrm{P}_{C} h_{m_{j}}-h_{m_{j}}, P_{C} h_{m_{j}}-z_{0}\right\rangle+\left\langle h_{m_{j}}-z_{0}, P_{C} h_{m_{j}}-z_{0}\right\rangle \\
& \leqslant\left\langle\alpha_{m_{j}} x_{0}+\beta_{m_{j}} y_{m_{j}}+\gamma_{m_{j}} z_{m_{j}}-z_{0}, x_{m_{j}+1}-z_{0}\right\rangle \\
& \leqslant\left\|\beta_{m_{j}}\left(y_{m_{j}}-z_{0}\right)+\gamma_{m_{j}}\left(z_{m_{j}}-z_{0}\right)\right\|\left\|x_{m_{j}+1}-z_{0}\right\|+\alpha_{m_{j}}\left\langle x_{0}-z_{0}, x_{m_{j}+1}-z_{0}\right\rangle \\
& \leqslant \beta_{m_{j}}\left\|z_{m_{j}}-z_{0}\right\|\left\|x_{m_{j}+1}-z_{0}\right\|+\gamma_{m_{j}}\left\|z_{m_{j}}-z_{0}\right\|\left\|x_{m_{j}+1}-z_{0}\right\|+\alpha_{m_{j}}\left\langle x_{0}-z_{0}, x_{m_{j}+1}-z_{0}\right\rangle \\
& \leqslant\left(1-\alpha_{m_{j}}\right)\left\|x_{m_{j}}-z_{0}\right\|\left\|x_{m_{j}+1}-z_{0}\right\|+\alpha_{m_{j}}\left\langle x_{0}-z_{0}, x_{m_{j}+1}-z_{0}\right\rangle \\
& \leqslant\left(1-\alpha_{m_{j}}\right)\left\|x_{m_{j}+1}-z_{0}\right\|^{2}+\alpha_{m_{j}}\left\langle x_{0}-z_{0}, x_{m_{j}}-z_{0}\right\rangle+\alpha_{m_{j}}\left\|x_{0}-z_{0}\right\|\left\|x_{m_{j}+1}-x_{m_{j}}\right\| .
\end{aligned}
$$


It follows that

$$
\left\|x_{m_{j}+1}-z_{0}\right\|^{2} \leqslant\left\langle x_{0}-z_{0}, x_{m_{j}}-z_{0}\right\rangle+\left\|x_{0}-z_{0}\right\|\left\|x_{m_{j}+1}-x_{m_{j}}\right\| .
$$

We deduce from (3.17), (3.18), and (3.19) that

$$
\lim _{j \rightarrow \infty}\left\|x_{m_{j}+1}-z_{0}\right\|=0 .
$$

Applying Lemma 2.2, we obtain

$$
0 \leqslant\left\|x_{j}-z_{0}\right\| \leqslant\left\|x_{m_{j}+1}-z_{0}\right\| .
$$

Consequently, we get $x_{n} \rightarrow z_{0}$ as $n \rightarrow \infty$. The proof is completed.

\section{An extension of our main results}

By using Theorem 3.4, we have the following strong convergence results for computing the common solution of fixed point problems of nonlinear mappings and generalized split feasibility problems in Banach spaces.

Conclusion 4.1. Let $\mathrm{E}$ be a smooth, strictly convex, and reflexive Banach space and let $\mathrm{J}$ be the duality mapping on $\mathrm{E}$. Let $\mathrm{H}$ be a Hilbert space and let $\mathrm{C}$ be a nonempty closed convex subset of $\mathrm{H}$. Let $\mathrm{A}$ be a maximal monotone operator of $\mathrm{H}$ into $2^{\mathrm{H}}$ such that $\operatorname{dom}(\mathrm{A}) \subset \mathrm{C}$. Let $\mathrm{J}_{\lambda}$ be the resolvent of $\mathrm{A}$ for $\lambda>0$ and let $\mathrm{T}$ be a firmly nonexpansive-like mapping on $\mathrm{E}$. Let $\mathrm{S}: \mathrm{H} \rightarrow \mathrm{E}$ be a bounded linear operator such that $\mathrm{S} \neq 0$ and let $\mathrm{S}^{*}$ be the adjoint operator of $\mathrm{S}$. Let $\mathrm{U}: \mathrm{C} \rightarrow \mathrm{C}$ be a 2-generalized hybrid mapping. Assume $\Gamma:=\mathrm{F}(\mathrm{U}) \cap \mathrm{A}^{-1} 0 \cap \mathrm{S}^{-1} \mathrm{~F}(\mathrm{~T})$ is nonempty and the element $x_{0} \in \mathrm{C}$ is fixed. Let $\left\{x_{n}\right\}$ be a sequence generated by

$$
\left\{\begin{array}{l}
x_{1} \text { chosen arbitrarily, } \\
z_{n}=J_{\lambda_{n}}\left(x_{n}-\delta_{n} S^{*} J\left(S x_{n}-T S x_{n}\right)\right), \\
y_{n}=\left(1-\sigma_{n}\right) z_{n}+\frac{\sigma_{n}}{n} \sum_{k=0}^{n-1} U^{k} z_{n}, \\
x_{n+1}=P_{C}\left(\alpha_{n} x_{0}+\beta_{n} y_{n}+\gamma_{n} z_{n}\right), \quad \forall n \in \mathbb{N},
\end{array}\right.
$$

where $\left\{\alpha_{n}\right\},\left\{\beta_{n}\right\},\left\{\gamma_{n}\right\} \subset(0,1)$ and $\left\{\lambda_{n}\right\},\left\{\delta_{n}\right\},\left\{\sigma_{n}\right\} \subset(0,+\infty)$ satisfy the following conditions:

(i) $\lim _{n \rightarrow \infty} \alpha_{n}=0$ and $\sum_{n=1}^{\infty} \alpha_{n}=\infty$;

(ii) $0<\liminf _{n \rightarrow \infty} \beta_{n} \leqslant \limsup _{n \rightarrow \infty} \beta_{n}<1$ and $\alpha_{n}+\beta_{n}+\gamma_{n}=1$;

(iii) $0<\mathrm{a} \leqslant \sigma_{\mathrm{n}} \leqslant \mathrm{b}<1$;

(iv) $0<\mathrm{c} \leqslant \delta_{\mathrm{n}} \leqslant \mathrm{d}<\frac{2}{\|\mathrm{~S}\|^{2}}$ and $0<\liminf _{\mathrm{n} \rightarrow \infty} \lambda_{\mathrm{n}} \leqslant \limsup _{\mathrm{n} \rightarrow \infty} \lambda_{\mathrm{n}}<\infty$.

Then the sequence $\left\{x_{n}\right\}$ generated by (4.1) converges strongly to a point $z_{0} \in \Gamma$, where $z_{0}=P_{\Gamma} x_{0}$.

Proof. It is easy to see that a 2-generalized hybrid mapping is 0-demimetric. In the same way as Theorem 3.4, we need only prove that when $x_{n_{i}} \rightarrow \tilde{x} \in C$, then $\tilde{x} \in F(U)$. Indeed, since $U$ is a 2-generalized hybrid mapping, there exist $\delta_{1}, \delta_{2}, \epsilon_{1}, \epsilon_{2} \in \mathbb{R}$ such that

$$
\begin{aligned}
& \delta_{1}\left\|\mathrm{u}^{2} x-\mathrm{u} y\right\|^{2}+\delta_{2}\|\mathrm{u} x-\mathrm{u} y\|^{2}+\left(1-\delta_{1}-\delta_{2}\right)\|x-\mathrm{u} y\|^{2} \\
& \leqslant \epsilon_{1}\left\|\mathrm{u}^{2} x-y\right\|^{2}+\epsilon_{2}\|\mathrm{u} x-y\|^{2}+\left(1-\epsilon_{1}-\epsilon_{2}\right)\|x-y\|^{2}
\end{aligned}
$$

for all $x, y \in C$. Replacing $x$ by $U^{k} z_{n}$ in above inequality, we have for all $y \in C$ and $k=0,1,2, \ldots, n-1$,

$$
\begin{aligned}
& \delta_{1}\left\|\mathrm{u}^{\mathrm{k}+2} z_{\mathrm{n}}-\mathrm{u} y\right\|^{2}+\delta_{2}\left\|\mathrm{u}^{\mathrm{k}+1} z_{\mathrm{n}}-\mathrm{u} y\right\|^{2}+\left(1-\delta_{1}-\delta_{2}\right)\left\|\mathrm{u}^{\mathrm{k}} z_{\mathrm{n}}-\mathrm{u} y\right\|^{2} \\
& \leqslant \epsilon_{1}\left\|\mathrm{u}^{\mathrm{k}+2} z_{\mathrm{n}}-\mathrm{y}\right\|^{2}+\epsilon_{2}\left\|\mathrm{u}^{\mathrm{k}+1} z_{\mathrm{n}}-\mathrm{y}\right\|^{2}+\left(1-\epsilon_{1}-\epsilon_{2}\right)\left\|\mathrm{u}^{\mathrm{k}} z_{\mathrm{n}}-\mathrm{y}\right\|^{2} \\
& \leqslant \epsilon_{1}\left(\left\|\mathrm{u}^{\mathrm{k}+2} z_{\mathrm{n}}-\mathrm{u} y\right\|^{2}+\|\mathrm{u} y-\mathrm{y}\|^{2}+2\left\langle\mathrm{u}^{\mathrm{k}+2} z_{\mathrm{n}}-\mathrm{u} \mathrm{y}, \mathrm{u} y-\mathrm{y}\right\rangle\right)
\end{aligned}
$$




$$
\begin{aligned}
& +\epsilon_{2}\left(\left\|\mathrm{u}^{\mathrm{k}+1} z_{\mathrm{n}}-\mathrm{u} y\right\|^{2}+\|\mathrm{u} y-\mathrm{y}\|^{2}+2\left\langle\mathrm{u}^{\mathrm{k}+1} z_{\mathrm{n}}-\mathrm{u} \mathrm{y}, \mathrm{U} y-\mathrm{y}\right\rangle\right) \\
& +\left(1-\epsilon_{1}-\epsilon_{2}\right)\left(\left\|\mathrm{u}^{\mathrm{k}} z_{\mathrm{n}}-\mathrm{u} y\right\|^{2}+\|\mathrm{u} y-\mathrm{y}\|^{2}+2\left\langle\mathrm{u}^{\mathrm{k}} z_{\mathrm{n}}-\mathrm{u} y, \mathrm{U} y-\mathrm{y}\right\rangle\right) .
\end{aligned}
$$

This implies that

$$
\begin{aligned}
& 0 \leqslant\left(\epsilon_{1}-\delta_{1}\right)\left\|\mathrm{U}^{\mathrm{k}+2} z_{\mathrm{n}}-\mathrm{Uy}\right\|^{2}+\|\mathrm{U} y-\mathrm{y}\|^{2}+2 \epsilon_{1}\left\langle\mathrm{u}^{\mathrm{k}+2} z_{\mathrm{n}}-\mathrm{Uy}, \mathrm{U} y-\mathrm{y}\right\rangle \\
& +\left(\epsilon_{2}-\delta_{2}\right)\left\|\mathrm{u}^{\mathrm{k}+1} z_{\mathrm{n}}-\mathrm{Uy}\right\|^{2}+2 \epsilon_{2}\left\langle\mathrm{u}^{\mathrm{k}+1} z_{\mathrm{n}}-\mathrm{Uy}, \mathrm{Uy}-\mathrm{y}\right\rangle \\
& +\left(\delta_{1}-\epsilon_{1}+\delta_{2}-\epsilon_{2}\right)\left\|\mathrm{u}^{\mathrm{k}} z_{\mathrm{n}}-\mathrm{Uy}\right\|^{2}+2\left(1-\epsilon_{1}-\epsilon_{2}\right)\left\langle\mathrm{u}^{\mathrm{k}} z_{\mathrm{n}}-\mathrm{Uy}, \mathrm{Uy}-\mathrm{y}\right\rangle \\
& \leqslant\left(\epsilon_{1}-\delta_{1}\right)\left(\left\|\mathrm{u}^{\mathrm{k}+2} z_{\mathrm{n}}-\mathrm{U} y\right\|^{2}-\left\|\mathrm{u}^{\mathrm{k}} z_{\mathrm{n}}-\mathrm{u} y\right\|^{2}\right)+\left(\epsilon_{2}-\delta_{2}\right)\left(\left\|\mathrm{u}^{\mathrm{k}+1} z_{\mathrm{n}}-\mathrm{uy}\right\|^{2}-\left\|\mathrm{u}^{\mathrm{k}} z_{\mathrm{n}}-\mathrm{u} y\right\|^{2}\right) \\
& +\|\mathrm{U} y-y\|^{2}+2\left\langle\mathrm{u}^{\mathrm{k}} z_{\mathfrak{n}}-\mathrm{Uy}+\epsilon_{1}\left(\mathrm{u}^{\mathrm{k}+2} z_{\mathfrak{n}}-\mathrm{u}^{\mathrm{k}} z_{\mathfrak{n}}\right)+\epsilon_{2}\left(\mathrm{U}^{\mathrm{k}+1} z_{\mathfrak{n}}-\mathrm{U}^{\mathrm{k}} z_{\mathfrak{n}}\right), \mathrm{U} y-\mathrm{y}\right\rangle .
\end{aligned}
$$

Set $U_{n}:=\frac{1}{n} \sum_{k=0}^{n-1} U^{k}$. Summing up these inequalities (4.2) with respect to $k=0$ to $k=n-1$ and dividing by $n$, we have

$$
\begin{aligned}
0 \leqslant & \frac{\epsilon_{1}-\delta_{1}}{n}\left(\left\|u^{n+1} z_{n}-U y\right\|^{2}+\left\|u^{n} z_{n}-U y\right\|^{2}-\left\|U z_{n}-U y\right\|^{2}-\left\|z_{n}-U y\right\|^{2}\right) \\
& +\frac{\epsilon_{2}-\delta_{2}}{n}\left(\left\|u^{n} z_{n}-U y\right\|^{2}-\left\|z_{n}-U y\right\|^{2}\right)+\|U y-y\|^{2}+2\left\langle u_{n} z_{n}-U y, U y-y\right\rangle \\
& +\frac{2}{n}\left\langle\epsilon_{1}\left(u^{n+1} z_{n}+U^{n} z_{n}-U z_{n}-z_{n}\right)+\epsilon_{2}\left(u^{n} z_{n}-z_{n}\right), U y-y\right\rangle .
\end{aligned}
$$

By the definition of the 2-generalized hybrid mapping, we find for all $x \in C$ and $x^{*} \in F(U)$ that

$$
\left\|x^{*}-u^{n} x\right\| \leqslant\left\|x^{*}-x\right\|, \quad \forall n \in \mathbb{N},
$$

which means that the sequence $\left\{U^{n} x\right\}$ is bounded for all $x \in C$ and $n \in \mathbb{N}$. On the other hand, following a similar argument as in Theorem 3.4, we get $\lim _{n \rightarrow \infty}\left\|z_{n}-x_{n}\right\|=0$ and $\lim _{n \rightarrow \infty}\left\|y_{n}-z_{n}\right\|=0$. Hence we deduce that $\lim _{n \rightarrow \infty}\left\|U_{n} z_{n}-z_{n}\right\|=0$. Replacing $n$ by $n_{i}$ and letting $n_{i} \rightarrow \infty$, we have from (4.3) that

$$
0 \leqslant\|\mathrm{U} y-y\|^{2}+2\langle\tilde{x}-\mathrm{Uy}, \mathrm{Uy}-\mathrm{y}\rangle \text {. }
$$

Taking $y=\tilde{x}$ in the above inequality, we have

$$
0 \leqslant\|\mathrm{U} \tilde{x}-\tilde{x}\|^{2}+2\langle\tilde{x}-\mathrm{U} \tilde{x}, \mathrm{U} \tilde{x}-\tilde{x}\rangle=\|\mathrm{U} \tilde{x}-\tilde{x}\|^{2}-2\|\mathrm{U} \tilde{x}-\tilde{x}\|^{2}=-\|\mathrm{U} \tilde{x}-\tilde{x}\|^{2} .
$$

This implies that $\tilde{x} \in F(U)$. The rest proof is similar to the proof of Theorem 3.4.

Conclusion 4.2. Let $\mathrm{E}$ be a smooth, strictly convex, and reflexive Banach space and let $\mathrm{J}$ be the duality mapping on $\mathrm{E}$. Let $\mathrm{H}$ be a Hilbert space and let $\mathrm{C}$ be a nonempty closed convex subset of $\mathrm{H}$. Let $\mathrm{A}$ be a maximal monotone operator of $\mathrm{H}$ into $2^{\mathrm{H}}$ such that $\operatorname{dom}(\mathrm{A}) \subset \mathrm{C}$. Let $\mathrm{J}_{\lambda}$ be the resolvent of $\mathrm{A}$ for $\lambda>0$ and let $\mathrm{T}$ be a firmly nonexpansive-like mapping on $\mathrm{E}$. Let $\mathrm{S}: \mathrm{H} \rightarrow \mathrm{E}$ be a bounded linear operator such that $\mathrm{S} \neq 0$ and let $\mathrm{S}^{*}$ be the adjoint operator of $\mathrm{S}$. Let $\left\{\mathrm{U}_{i}\right\}_{i=1}^{\infty}: \mathrm{C} \rightarrow \mathrm{H}$ be an infinite family of generalized hybrid mappings. Assume $\Gamma:=\bigcap_{i=1}^{\infty} \mathrm{F}\left(\mathrm{U}_{\mathrm{i}}\right) \cap A^{-1} 0 \bigcap \mathrm{S}^{-1} \mathrm{~F}(\mathrm{~T})$ is nonempty and the element $\mathrm{x}_{0} \in \mathrm{C}$ is fixed. Let $\left\{\mathrm{x}_{\mathrm{n}}\right\}$ be a sequence generated by

$$
\left\{\begin{array}{l}
x_{1} \text { chosen arbitrarily, } \\
z_{n}=J_{\lambda_{n}}\left(x_{n}-\delta_{n} S^{*} J\left(S x_{n}-T S x_{n}\right)\right), \\
y_{n}=\left(1-\sigma_{n}\right) z_{n}+\sigma_{n} \sum_{i=1}^{\infty} \eta_{i} U_{i} z_{n}, \\
x_{n+1}=P_{C}\left(\alpha_{n} x_{0}+\beta_{n} y_{n}+\gamma_{n} z_{n}\right), \quad \forall n \in \mathbb{N},
\end{array}\right.
$$

where $\left\{\alpha_{n}\right\},\left\{\beta_{n}\right\},\left\{\gamma_{n}\right\},\left\{\eta_{i}\right\} \subset(0,1)$ and $\left\{\lambda_{n}\right\},\left\{\delta_{n}\right\},\left\{\sigma_{n}\right\} \subset(0,+\infty)$ satisfy the following conditions:

(i) $\lim _{n \rightarrow \infty} \alpha_{n}=0$ and $\sum_{n=1}^{\infty} \alpha_{n}=\infty$; 
(ii) $0<\liminf _{n \rightarrow \infty} \beta_{n} \leqslant \limsup _{n \rightarrow \infty} \beta_{n}<1$ and $\alpha_{n}+\beta_{n}+\gamma_{n}=1$;

(iii) $\sum_{i=1}^{\infty} \eta_{\mathfrak{i}}=1$ and $0<a \leqslant \sigma_{\mathfrak{n}} \leqslant b<1$;

(iv) $0<\mathrm{c} \leqslant \delta_{\mathrm{n}} \leqslant \mathrm{d}<\frac{2}{\|S\|^{2}}$ and $0<\liminf _{\mathrm{n} \rightarrow \infty} \lambda_{\mathrm{n}} \leqslant \limsup _{\mathrm{n} \rightarrow \infty} \lambda_{\mathrm{n}}<\infty$.

Then the sequence $\left\{x_{n}\right\}$ generated by (4.4) converges strongly to a point $z_{0} \in \Gamma$, where $z_{0}=\mathrm{P}_{\Gamma} x_{0}$.

Proof. We know that the generalized hybrid mapping is 0-demimetric and demiclosed; see [13]. Therefore, Theorem 3.4 implies the conclusion.

\section{Applications}

Using Theorem 3.4, we first study the problem of approximating zero points of maximal monotone operators.

Theorem 5.1. Let $\mathrm{E}$ be a smooth, strictly convex, and reflexive Banach space and let $\mathrm{J}$ be the duality mapping on $\mathrm{E}$. Let $\mathrm{H}$ be a Hilbert space and let $\mathrm{C}$ be a nonempty closed convex subset of $\mathrm{H}$. Let $\mathrm{A}$ and $\mathrm{B}$ be maximal monotone operators of $\mathrm{H}$ into $2^{\mathrm{H}}$ such that $\operatorname{dom}(\mathrm{A}) \subset \mathrm{C}$ and $\mathrm{E}$ into $2^{\mathrm{E}}$, respectively. Let $\mathrm{J}_{\lambda}$ be the resolvent of $\mathrm{A}$ for $\lambda>0$ and let $\mathrm{Q}_{\mu}$ be the metric resolvent of $\mathrm{B}$ for $\mu>0$. Let $\mathrm{S}: \mathrm{H} \rightarrow \mathrm{E}$ be a bounded linear operator such that $\mathrm{S} \neq 0$ and let $\mathrm{S}^{*}$ be the adjoint operator of $\mathrm{S}$. Let $\left\{\mathrm{U}_{i}\right\}_{i=1}^{\infty}: \mathrm{C} \rightarrow \mathrm{H}$ be an infinite family of $\mathrm{k}_{\mathrm{i}}$-demimetric and demiclosed mappings. Assume $\Gamma:=\bigcap_{i=1}^{\infty} \mathrm{F}\left(\mathrm{U}_{i}\right) \cap A^{-1} 0 \bigcap S^{-1}\left(\mathrm{~B}^{-1} 0\right)$ is nonempty and the element $\mathrm{x}_{0} \in \mathrm{C}$ is fixed. Let $\left\{\mathrm{x}_{\mathrm{n}}\right\}$ be a sequence generated by

$$
\left\{\begin{array}{l}
x_{1} \quad \text { chosen arbitrarily, } \\
z_{n}=J_{\lambda_{n}}\left(x_{n}-\delta_{n} S^{*} J\left(S x_{n}-Q_{\mu} S x_{n}\right)\right), \\
y_{n}=\left(1-\sigma_{n}\right) z_{n}+\sigma_{n} \sum_{i=1}^{\infty} \eta_{i} U_{i} z_{n}, \\
x_{n+1}=P_{C}\left(\alpha_{n} x_{0}+\beta_{n} y_{n}+\gamma_{n} z_{n}\right), \quad \forall n \in \mathbb{N}
\end{array}\right.
$$

where $\left\{\alpha_{n}\right\},\left\{\beta_{n}\right\},\left\{\gamma_{n}\right\},\left\{\eta_{i}\right\} \subset(0,1)$ and $\left\{\lambda_{n}\right\},\left\{\delta_{n}\right\},\left\{\sigma_{n}\right\} \subset(0,+\infty)$ satisfy the following conditions:

(i) $\lim _{n \rightarrow \infty} \alpha_{n}=0$ and $\sum_{n=1}^{\infty} \alpha_{n}=\infty$;

(ii) $0<\liminf _{n \rightarrow \infty} \beta_{n} \leqslant \limsup _{n \rightarrow \infty} \beta_{n}<1$ and $\alpha_{n}+\beta_{n}+\gamma_{n}=1$;

(iii) $\sum_{i=1}^{\infty} \eta_{i}=1$;

(iv) $0<a \leqslant \sigma_{n} \leqslant b<1-k$ and $k=\sup \left\{k_{i}: i \in \mathbb{N}\right\}<1$;

(v) $0<\mathrm{c} \leqslant \delta_{\mathrm{n}} \leqslant \mathrm{d}<\frac{2}{\|S\|^{2}}$ and $0<\liminf _{n \rightarrow \infty} \lambda_{n} \leqslant \limsup _{n \rightarrow \infty} \lambda_{n}<\infty$.

Then the sequence $\left\{x_{n}\right\}$ generated by (5.1) converges strongly to a point $z_{0} \in \Gamma$, where $z_{0}=P_{\Gamma} x_{0}$.

Proof. It is well known that each $Q_{\mu}$ is a single valued mapping of $E$ into itself and $F\left(Q_{\mu}\right)=B^{-1} 0$; see $[4,20]$. We also know that each $Q_{\mu}$ is firmly nonexpansive-like; see $[3,4]$. Therefore, Theorem 3.4 implies the Theorem 5.1.

Using Theorem 5.1, we next study the problem of minimizing a convex function. For a Banach space $E$ and a function $f: E \rightarrow(-\infty, \infty]$, we denote by $\partial f$ the subdifferential of $f$ defined by

$$
\partial f(x)=\left\{x^{*} \in E^{*}:\left(f-x^{*}\right)(x)=\inf \left(f-x^{*}\right)(E)\right\}
$$

for all $x \in E$.

Theorem 5.2. Let $\mathrm{E}$ be a smooth, strictly convex, and reflexive Banach space and let $\mathrm{J}$ be the duality mapping on $\mathrm{E}$. Let $\mathrm{H}$ be a Hilbert space and let $\mathrm{C}$ be a nonempty closed convex subset of $\mathrm{H}$. Let $\mathrm{f}: \mathrm{E} \rightarrow \mathbb{R}$ be a proper, convex and lower semicontinuous function such that $\partial \mathrm{f}^{-1}(0) \neq \emptyset$. Let $\mathrm{A}$ be a maximal monotone operator of $\mathrm{H}$ into $2^{\mathrm{H}}$ such that $\operatorname{dom}(\mathrm{A}) \subset \mathrm{C}$. Let $\mathrm{J}_{\lambda}$ be the resolvent of $\mathrm{A}$ for $\lambda>0$. Let $\mathrm{S}: \mathrm{H} \rightarrow \mathrm{E}$ be a bounded linear operator such that $\mathrm{S} \neq 0$ and let $\mathrm{S}^{*}$ be the adjoint operator of $\mathrm{S}$. Let $\left\{\mathrm{U}_{i}\right\}_{i=1}^{\infty}: \mathrm{C} \rightarrow \mathrm{H}$ be an infinite family of $\mathrm{k}_{\mathrm{i}}$-demimetric and 
demiclosed mappings. Assume $\Gamma:=\bigcap_{i=1}^{\infty} \mathrm{F}\left(\mathrm{U}_{i}\right) \bigcap \mathrm{A}^{-1} 0 \bigcap \mathrm{S}^{-1}\left(\partial \mathrm{f}^{-1}(0)\right)$ is nonempty and the element $\mathrm{x}_{0} \in \mathrm{C}$ is fixed. Let $\left\{x_{n}\right\}$ be a sequence generated by

$$
\left\{\begin{array}{l}
x_{1} \text { chosen arbitrarily, } \\
w_{n}=\arg \min _{y \in E}\left\{f(y)+(2 \mu)^{-1}\left\|y-S x_{n}\right\|^{2}\right\} \\
z_{n}=J_{\lambda_{n}}\left(x_{n}-\delta_{n} S^{*} J\left(S x_{n}-w_{n}\right)\right), \\
y_{n}=\left(1-\sigma_{n}\right) z_{n}+\sigma_{n} \sum_{i=1}^{\infty} \eta_{i} U_{i} z_{n}, \\
x_{n+1}=P_{C}\left(\alpha_{n} x_{0}+\beta_{n} y_{n}+\gamma_{n} z_{n}\right), \quad \forall n \in \mathbb{N},
\end{array}\right.
$$

where $\left\{\alpha_{n}\right\},\left\{\beta_{n}\right\},\left\{\gamma_{n}\right\},\left\{\eta_{i}\right\} \subset(0,1), \mu \in(0,+\infty)$ and $\left\{\lambda_{n}\right\},\left\{\delta_{n}\right\},\left\{\sigma_{n}\right\} \subset(0,+\infty)$ satisfy the following conditions:

(i) $\lim _{n \rightarrow \infty} \alpha_{n}=0$ and $\sum_{n=1}^{\infty} \alpha_{n}=\infty$;

(ii) $0<\liminf _{n \rightarrow \infty} \beta_{n} \leqslant \limsup _{n \rightarrow \infty} \beta_{n}<1$ and $\alpha_{n}+\beta_{n}+\gamma_{n}=1$;

(iii) $\sum_{i=1}^{\infty} \eta_{i}=1$;

(iv) $0<\mathrm{a} \leqslant \sigma_{\mathrm{n}} \leqslant \mathrm{b}<1-\mathrm{k}$ and $\mathrm{k}=\sup \left\{\mathrm{k}_{\mathrm{i}}: \mathrm{i} \in \mathbb{N}\right\}<1$;

(v) $0<\mathrm{c} \leqslant \delta_{\mathrm{n}} \leqslant \mathrm{d}<\frac{2}{\|\mathrm{~s}\|^{2}}$ and $0<\liminf _{\mathrm{n} \rightarrow \infty} \lambda_{\mathrm{n}} \leqslant \limsup _{\mathrm{n} \rightarrow \infty} \lambda_{\mathrm{n}}<\infty$.

Then the sequence $\left\{x_{n}\right\}$ generated by (5.2) converges strongly to a point $z_{0} \in \Gamma$, where $z_{0}=P_{\Gamma} x_{0}$.

Proof. We know that $\partial f: E \rightarrow E^{*}$ is maximal monotone and $(\partial f)^{-1}(0)=\arg \min f$; see $[19,20]$. We also know that

$$
\left(I+\mu J^{-1} \partial f\right)^{-1}(x)=\arg \min _{y \in E}\left\{f(y)+(2 \mu)^{-1}\|y-x\|^{2}\right\}
$$

for all $\mu>0$ and $x \in E$, where I denotes the identity mapping on E. Therefore, we obtain the desired result.

Let $\mathrm{H}$ be a Hilbert space and let $\mathrm{g}: \mathrm{H} \rightarrow(-\infty,+\infty]$ be a proper convex lower semi-continuous function. Then, the subdifferential $\partial \mathrm{g}$ of $\mathrm{g}$ is defined as follows:

$$
\partial g=\{y \in H: g(z) \geqslant g(x)+\langle z-x, y\rangle, z \in H\}, \quad \forall x \in H .
$$

From Rockafellar [21], we know that $\partial g$ is maximal monotone. It is easy to verify that $0 \in \partial g(x)$ if and only if $g(x)=\min _{y \in H} g(y)$. Let $I_{C}$ be the indicator function of $C$, i.e.,

$$
\mathrm{I}_{\mathrm{C}}(x)= \begin{cases}0, & x \in \mathrm{C} \\ +\infty, & x \notin \mathrm{C}\end{cases}
$$

Then, $I_{C}$ is a proper lower semi-continuous convex function on $H$, and the subdifferential $\partial I_{C}$ of $I_{C}$ is a maximal monotone operator. Furthermore, suppose $\mathrm{C}$ is a nonempty closed convex subset. Then,

$$
\left(\mathrm{I}+\lambda \partial \mathrm{I}_{\mathrm{C}}\right)^{-1} \mathrm{x}=\mathrm{P}_{\mathrm{C}} \mathrm{x}, \quad \forall x \in \mathrm{H}, \lambda>0 .
$$

For more details, one can refer to [26].

Applying Theorem 3.4 to the case where $A=\partial \mathrm{I}_{C}$, we have the following result.

Theorem 5.3. Let $\mathrm{E}$ be a smooth, strictly convex, and reflexive Banach space and let $\mathrm{J}$ be the duality mapping on $\mathrm{E}$. Let $\mathrm{H}$ be a Hilbert space and let $\mathrm{C}$ be a nonempty closed convex subset of $\mathrm{H}$. Let $\mathrm{T}$ be a firmly nonexpansivelike mapping on $\mathrm{E}$. Let $\mathrm{S}: \mathrm{H} \rightarrow \mathrm{E}$ be a bounded linear operator such that $\mathrm{S} \neq 0$ and let $\mathrm{S}^{*}$ be the adjoint operator of $\mathrm{S}$. Let $\left\{\mathrm{U}_{i}\right\}_{i=1}^{\infty}: \mathrm{C} \rightarrow \mathrm{H}$ be an infinite family of $\mathrm{k}_{\mathrm{i}}$-demimetric and demiclosed mappings. Assume $\Gamma:=\bigcap_{i=1}^{\infty} \mathrm{F}\left(\mathrm{U}_{\mathrm{i}}\right) \cap \mathrm{S}^{-1} \mathrm{~F}(\mathrm{~T})$ is nonempty and the element $\mathrm{x}_{0} \in \mathrm{C}$ is fixed. Let $\left\{\mathrm{x}_{\mathrm{n}}\right\}$ be a sequence generated by

$$
\left\{\begin{array}{l}
x_{1} \text { chosen arbitrarily, } \\
z_{n}=P_{C}\left(x_{n}-\delta_{n} S^{*} J\left(S x_{n}-T S x_{n}\right)\right), \\
y_{n}=\left(1-\sigma_{n}\right) z_{n}+\sigma_{n} \sum_{i=1}^{\infty} \eta_{i} U_{i} z_{n}, \\
x_{n+1}=P_{C}\left(\alpha_{n} x_{0}+\beta_{n} y_{n}+\gamma_{n} z_{n}\right), \quad \forall n \in \mathbb{N},
\end{array}\right.
$$

where $\left\{\alpha_{n}\right\},\left\{\beta_{n}\right\},\left\{\gamma_{n}\right\},\left\{\eta_{i}\right\} \subset(0,1)$ and $\left\{\delta_{n}\right\},\left\{\sigma_{n}\right\} \subset(0,+\infty)$ satisfy the following conditions: 
(i) $\lim _{n \rightarrow \infty} \alpha_{n}=0$ and $\sum_{n=1}^{\infty} \alpha_{n}=\infty$;

(ii) $0<\liminf _{n \rightarrow \infty} \beta_{n} \leqslant \limsup _{n \rightarrow \infty} \beta_{n}<1$ and $\alpha_{n}+\beta_{n}+\gamma_{n}=1$;

(iii) $\sum_{i=1}^{\infty} \eta_{i}=1$;

(iv) $0<\mathrm{a} \leqslant \sigma_{\mathrm{n}} \leqslant \mathrm{b}<1-\mathrm{k}$ and $\mathrm{k}=\sup \left\{\mathrm{k}_{\mathrm{i}}: \mathrm{i} \in \mathbb{N}\right\}<1$;

(v) $0<\mathrm{c} \leqslant \delta_{\mathrm{n}} \leqslant \mathrm{d}<\frac{2}{\|S\|^{2}}$.

Then the sequence $\left\{x_{n}\right\}$ generated by (5.3) converges strongly to a point $z_{0} \in \Gamma$, where $z_{0}=\mathrm{P}_{\Gamma} x_{0}$.

\section{Numerical examples}

The purpose of this section is to give two numerical examples supporting Theorem 3.4.

Table 1: The values of the sequence $\left\{x_{n}\right\}$.

\begin{tabular}{cccccc}
\hline $\mathrm{n}$ & & \multicolumn{4}{c}{$\mathrm{x}_{\mathrm{n}}$} \\
\hline $1-5$ & 1.000000000000 & 0.136458333333 & 0.019509412977 & 0.007067027004 & 0.004703615999 \\
$6-10$ & 0.003687224442 & 0.003053557087 & 0.002608427454 & 0.002277089072 & 0.002020626483 \\
$\vdots$ & $\vdots$ & $\vdots$ & $\vdots$ & $\vdots$ & $\vdots$ \\
$21-25$ & 0.000903174745 & 0.000859959418 & 0.000820691798 & 0.000784854452 & 0.000752016612 \\
$\vdots$ & $\vdots$ & $\vdots$ & $\vdots$ & $\vdots$ & $\vdots$ \\
$46-50$ & 0.000400320289 & 0.000391599776 & 0.000383251114 & 0.000375251014 & 0.000367578092 \\
\hline
\end{tabular}

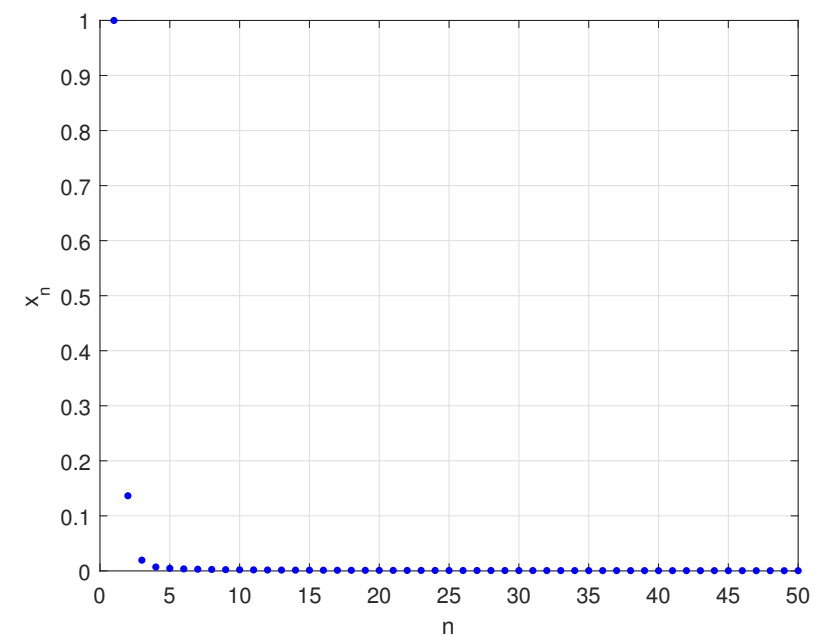

Figure 1: The convergence of $\left\{x_{n}\right\}$ with initial values $x_{1}=1$.

Example 6.1. Let $\mathrm{C}=\mathrm{H}=\mathbb{R}$ with the inner product defined by $\langle x, y\rangle=x y$ for all $x, y \in \mathbb{R}$ and the standard norm $|\cdot|$. Letting $S: H \rightarrow E$ be defined as $S x=-\frac{3}{2} x$ for all $x \in H$, we then see $S$ is a bounded linear operator with its adjoint $S^{*}=S$. Let $T: E \rightarrow E$ be defined as $T x=\frac{1}{2} x$ for all $x \in E, A: H \rightarrow H$ be defined as $A x=3 x$ for all $x \in H$, and $U_{i}: H \rightarrow H$ be defined as $U_{i} x=-2 x$ for all $i \in \mathbb{N}$ and $x \in H$. It is easy to check that $\Gamma=\{0\}$. Also, it is easy to check $T$ is firmly nonexpansive-like, $A$ is maximal monotone, and $U_{i}$ are $\frac{1}{3}$-demimetric and demiclosed for all $i \in \mathbb{N}$.

Let us choose $\alpha_{n}=\frac{1}{6 n}, \beta_{n}=\frac{n+1}{3 n}, \gamma_{n}=\frac{4 n-3}{6 n}, \delta_{n}=\sigma_{n}=\frac{2 n-1}{4 n}, \lambda_{n}=\frac{2 n-1}{3 n}$, and $\eta_{i}=\frac{1}{2^{i}}$ for all $n, i \in \mathbb{N}$. Then $\alpha_{n}, \beta_{n}, \gamma_{n}, \lambda_{n}, \delta_{n}, \sigma_{n}, \eta_{i}$ satisfy all the conditions of Theorem 3.4. Therefore iterative 
scheme (3.3) becomes

$$
x_{n+1}=\frac{1}{6 n} x_{0}+\frac{-28 n^{3}-4 n^{2}+51 n+27}{448 n^{2}(3 n-1)} x_{n}+\frac{56 n^{2}-6 n-27}{192(3 n-1)} x_{n}, \quad \forall n \in \mathbb{N}
$$

Taking $x_{0}=0.1$ and $x_{1}=1$, we see that numerical results in Table 1 and Figure 1 demonstrate Theorem 3.4.

Example 6.2. Let $\mathrm{C}=\mathrm{H}=\ell_{2}^{3}$ and $\mathrm{E}=\ell_{3}^{3}$, where $\ell_{\mathrm{p}}^{n}$ denote the $\mathbb{R}^{\mathrm{n}}$ equipped with the norm $\|\mathbf{x}\|_{\mathrm{p}}=$ $\left(\sum_{i=1}^{n}\left|x^{(i)}\right|^{p}\right)^{1 / p}$ for $1 \leqslant p<\infty$. Then we know $H$ is a Hilbert space and $E$ is a smooth, strictly convex, and reflexive Banach space. As we know, in the $\ell_{3}^{n}$ space, the duality mapping is given by

$$
J \mathbf{x}= \begin{cases}\mathbf{0}, & \mathbf{x}=\mathbf{0}, \\ \left(x^{(1)}\left|x^{(1)}\right| /\|\mathbf{x}\|_{3}, x^{(2)}\left|x^{(2)}\right| /\|\mathbf{x}\|_{3}, \cdots, x^{(n)}\left|x^{(n)}\right| /\|\mathbf{x}\|_{3}\right), & \mathbf{x} \neq \mathbf{0} .\end{cases}
$$

Letting $S: H \rightarrow E$ be defined by $S \mathbf{x}=\frac{3}{2} \mathbf{x}$ for all $x \in H$, we then see $S$ is a bounded linear operator with its adjoint $S^{*}=S$. Let $T: E \rightarrow E$ be defined as $T \mathbf{x}=\frac{1}{2} \mathbf{x}$ for all $x \in E, A: H \rightarrow H$ be defined as $A \mathbf{x}=4 \mathbf{x}$ for all $x \in H$ and $U_{i}: H \rightarrow H$ be defined as $U_{i} \mathbf{x}=-\frac{1+i}{i} \mathbf{x}$ for all $i \in \mathbb{N}$ and $x \in H$. It is easy to check that $\Gamma=\{\mathbf{0}\}$. Also, it is easy to check $T$ is nonexpansive-like, $A$ is maximal monotone, and $U_{i}$ is $\frac{1}{1+2 i}$-demimetric and demiclosed for each $i \in \mathbb{N}$.

Let us choose $\alpha_{n}=\frac{1}{6 n}, \beta_{n}=\frac{n+1}{3 n}, \gamma_{n}=\frac{4 n-3}{6 n}, \delta_{n}=\sigma_{n}=\frac{2 n-1}{6 n}, \lambda_{n}=\frac{2 n-1}{4 n}$, and $\eta_{i}=\frac{1}{2^{i}}$ for all $n, i \in \mathbb{N}$. Then $\alpha_{n}, \beta_{n}, \gamma_{n}, \lambda_{n}, \delta_{n}, \sigma_{n}$, and $\eta_{i}$ satisfy all the conditions of Theorem 3.4. Hence iterative scheme (3.3) can be rewrite as

$$
\left\{\begin{array}{l}
\mathbf{z}_{\mathbf{n}}=\frac{n}{3 n-1}\left(\mathbf{x}_{\mathbf{n}}-\frac{6 n-3}{16 n} J \mathbf{x}_{\mathbf{n}}\right), \\
\mathbf{y}_{\mathbf{n}}=\frac{2 n+2-(2 n-1) \ln 2}{6 n} \mathbf{z}_{\mathbf{n}}, \\
\mathbf{x}_{\mathbf{n}+1}=\frac{1}{6 n} \mathbf{x}_{\mathbf{0}}+\frac{n+1}{3 n} \mathbf{y}_{\mathbf{n}}+\frac{4 n-3}{6 n} \mathbf{z}_{\mathbf{n}}, \quad \forall n \in \mathbb{N},
\end{array}\right.
$$

where

$$
J \mathbf{x}_{\mathbf{n}}= \begin{cases}\mathbf{0}, & \mathbf{x}_{\mathbf{n}}=\mathbf{0}, \\ \left(x^{(1)}\left|x^{(1)}\right| /\|\mathbf{x}\|_{3}, x^{(2)}\left|x^{(2)}\right| /\|\mathbf{x}\|_{3}, x^{(3)}\left|x^{(3)}\right| /\|\mathbf{x}\|_{3}\right), & \mathbf{x}_{\mathbf{n}} \neq \mathbf{0} .\end{cases}
$$

Using the algorithm (6.1) and taking $\mathbf{x}_{\mathbf{0}}=(0,0,0)$ and $\mathbf{x}_{\mathbf{1}}=(1,3,2)$, we report the numerical results in Table 2. In addition, Figure 2 also demonstrates Theorem 3.4.

Table 2: The values of the sequence $\left\{\mathbf{x}_{n}\right\}$.

\begin{tabular}{cccc}
\hline $\mathrm{n}$ & $\mathbf{x}_{\mathrm{n}}^{1}$ & $\mathbf{x}_{\mathrm{n}}^{2}$ & $\mathbf{x}_{n}^{3}$ \\
\hline 1 & 1.0000000000000 & 3.0000000000000 & 2.0000000000000 \\
2 & 0.2518830885020 & 0.6646635233715 & 0.4734375962925 \\
3 & 0.0528922212860 & 0.1155835401108 & 0.0902451087661 \\
4 & 0.0106450374252 & 0.0193955015251 & 0.0163638209461 \\
5 & 0.0020787650463 & 0.0032424586881 & 0.0028949700508 \\
$\vdots$ & $\vdots$ & $\vdots$ & $\vdots$ \\
10 & 0.0000004296960 & 0.0000004623849 & 0.0000004559153 \\
$\vdots$ & $\vdots$ & $\vdots$ & $\vdots$ \\
16 & $1.235753255427350 \times 10^{-11}$ & $1.243931512007411 \times 10^{-11}$ & $1.242438902187192 \times 10^{-11}$ \\
17 & $2.147941556198610 \times 10^{-12}$ & $2.157364622440542 \times 10^{-12}$ & $2.155649523624184 \times 10^{-12}$ \\
18 & $3.730736375573632 \times 10^{-13}$ & $3.741574065532121 \times 10^{-13}$ & $3.739605085662673 \times 10^{-13}$ \\
19 & $6.476321429387085 \times 10^{-14}$ & $6.488766725555732 \times 10^{-14}$ & $6.486508419914774 \times 10^{-14}$ \\
20 & $1.123777174154652 \times 10^{-14}$ & $1.125204375328997 \times 10^{-14}$ & $1.124945606067749 \times 10^{-14}$ \\
\hline
\end{tabular}




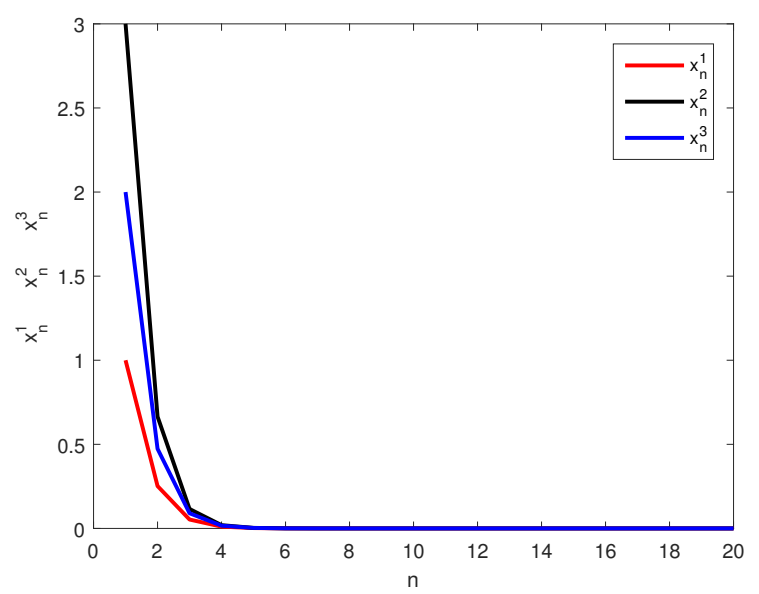

(a) $2 \mathrm{D}$

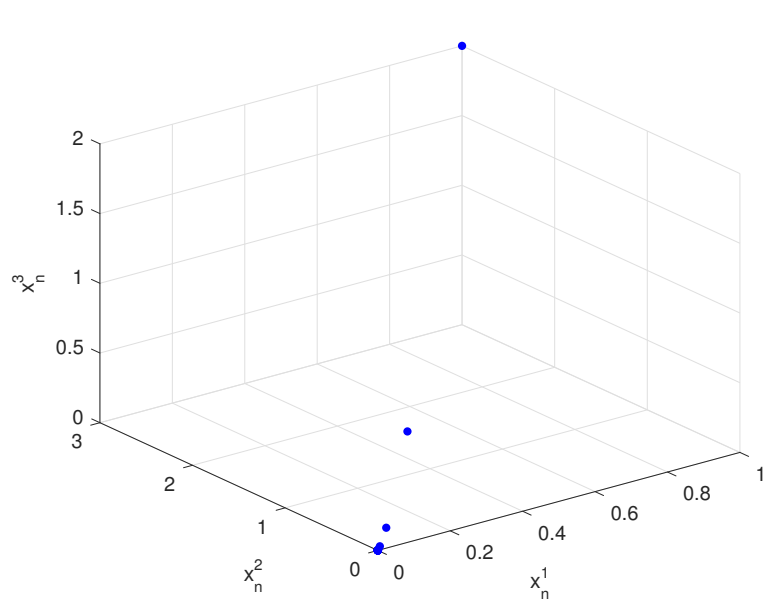

(b) 3D

Figure 2: The convergence of $\left\{\mathbf{x}_{n}\right\}$ with initial values $\mathbf{x}_{1}=(1,3,2)$.

\section{Acknowledgment}

This research was partially supported by the Innovation Program of Shanghai Municipal Education Commission (15ZZ068) and Ph.D. Research Foundation of Zhongyuan University of Technology (2200/34110323).

\section{References}

[1] S. Akashi, W. Takahashi, Weak convergence theorem for an infinite family of demimetric mappings in a Hilbert space, J. Nonlinear Convex Anal., 10 (2016), 2159-2169. 1, 2.5

[2] K. Aoyama, F. Kohsaka, Existence of fixed points of firmly nonexpansive-like mappings in Banach spaces, Fixed Point Theory Appl., 2010 (2010), 15 pages. 1

[3] K. Aoyama, F. Kohsaka, W. Takahashi, Strong convergence theorems for a family of mappings of type (P) and applications, Nonlinear Anal. Optim., 2009 (2009), 17 pages. 1, 2.4, 5

[4] K. Aoyama, F. Kohsaka, W. Takahashi, Three generalizations of firmly nonexpansive mappings: their relations and continuous properties, J. Nonlinear Convex Anal., 10 (2009), 131-147. 1, 1, 2, 5

[5] F. E. Browder, Nonlinear maximal monotone operators in Banach spaces, Math. Ann., 175 (1968), 89-113. 2

[6] C. Byrne, Iterative oblique projection onto convex sets and the split feasibility problem, Inverse Probl., 18 (2002), $441-453$. 1

[7] Y. Censor, T. Elfving, A multiprojection algorithm using Bregman projections in a product space, Numer. Algorithms, 8 (1994), 221-239. 1

[8] Y. Censor, A. Segal, The split common fxed point problem for directed operators, J. Convex Anal., 16 (2009), 587-600. 1

[9] S. S. Chang, L. Wang, Y. Zhao, On a class of split equality fixed point problems in Hilbert spaces, J. Nonlinear Var. Anal., 1 (2017), 201-212. 1

[10] C. Chidume, Ş. Măruster, Iterative methods for the computation of fixed points of demicontractive mappings, J. Comput. Appl. Math., 234 (2010), 861-882. 1.5

[11] S. Y. Cho, Strong convergence analysis of a hybrid algorithm for nonlinear operators in a Banach space, J. Appl. Anal. Comput., 8 (2018), 19-31. 1

[12] M. Hojo, W. Takahashi, I. Termwuttipong, Strong convergence theorems for 2-generalized hybrid mappings in Hilbert spaces, Nonlinear Anal., 75 (2012), 2166-2176. 1

[13] P. Kocourek, W. Takahashi, J.-C. Yao, Fixed point theorems and weak convergence theorems for generalized hybrid mappings in Hilbert spaces, Taiwanese J. Math., 14 (2010), 2497-2511. 1, 4.2

[14] P. E. Maingé, Approximation methods for common fixed points of nonexpansive mappings in Hilbert spaces, J. Math. Anal. Appl., 325 (2007), 469-479. 2.2

[15] G. Marino, H.-K. Xu, Weak and strong convergence theorems for strict pseudo-contractions in Hilbert spaces, J. Math. Anal. Appl., 329 (2007), 336-346. 1, 2.6

[16] S. Matsushita, W. Takahashi, Weak and strong convergence theorems for relatively nonexpansive mappings in Banach spaces, Fixed Point Theory Appl., 2004 (2004), 10 pages. 2 
[17] E. Naraghirad, L. J. Lin, Strong convergence theorems for generalized nonexpansive mappings on star-shaped set with applications, Fixed Point Theory Appl., 2014 (2014), 24 pages. 1.2

[18] X. Qin, J.-C. Yao, Projection splitting algorithm for nonself operator, J. Nonlinear Convex Anal., 18 (2017), $925-935.1$

[19] R. T. Rockafellar, Characterization of the subdifferentials of convex functions, Pacific J. Math., 17 (1966), 497-510. 5.2

[20] R. T. Rockafellar, On the maximality of sums of nonlinear monotone operators, Trans. Amer. Math. Soc., 149 (1970), 75-88. 5, 5.2

[21] R. T. Rockafellar, Augmented Lagrangians and applications of the proximal point algorithm in convex programming, Math. Oper. Res., 1 (1976), 97-116. 5

[22] Y. Shehu, O. S. Iyiola, C. D. Enyi, An iterative algorithm for solving split feasibility problems and fixed point problems in Banach spaces, Numer. Algorithms, 72 (2016), 835-864. 1

[23] W. Takahashi, Convex Analysis and Approximation of Fixed Points, Yokohama Publishers, Yokohama, (2000). 2

[24] W. Takahashi, Nonlinear Functional Analysis, Yokohama Publ., Yokohama, (2000). 2

[25] S. Takahashi, W. Takahashi, The split common null point problem and the shrinking projection method in Banach spaces, Optimization, 65 (2016), 281-287. 1

[26] S. Takahashi, W. Takahashi, M. Toyoda, Strong convergence theorems for maximal monotone operators with nonlinear mappings in Hilbert spaces, J. Optim. Theory Appl., 147 (2010), 27-41. 5

[27] W. Takahashi, J.-C. Yao, Strong convergence theorems by hybrid methods for the split common null point problem in Banach spaces, Fixed Point Theory Appl., 2015 (2015), 13 pages. 1

[28] K.-K. Tan, H.-K. Xu, Approximating fixed points of nonexpansive mappings by the Ishikawa iteration process, J. Math. Anal. Appl., 178 (1993), 301-308.

[29] H.-K. Xu, Iterative algorithms for nonlinear operators, J. London Math. Soc., 66 (2002), 240-256. 2.3

[30] Y.-H.Yao, H.-Y. Zhou, Y.-C. Liou, Weak and strong convergence theorems for an asymptotically $k$-strict pseudo-contraction and a mixed equilibrium problem, J. Korean Math. Soc., 46 (2009), 561-576. 1

[31] Z.-T. Yu, L.-J. Lin, C.-S. Chuang, Mathematical programming with multiple sets split monotone variational inclusion constraints, Fixed Point Theory Appl., 2014 (2014), 27 pages. 1 